\title{
On the roughness of the paths of RBM in a wedge
}

\author{
Peter Lakner ${ }^{\mathrm{a}}$, Josh Reed ${ }^{\mathrm{a}}$ and Bert Zwart ${ }^{\mathrm{b}}$ \\ ${ }^{a}$ Stern School of Business, New York University, 44 West 4th Street, New York, NY 10012, USA. \\ E-mail: plakner@stern.nyu.edu; jreed@stern.nyu.edu \\ b CWI, P.O. Box 94079, 1090 GB, Amsterdam, The Netherlands.E-mail: Bert.Zwart@cwi.nl
}

Received 15 June 2016; revised 30 July 2018; accepted 23 August 2018

\begin{abstract}
Reflected Brownian motion (RBM) in a wedge is a 2-dimensional stochastic process $Z$ whose state space in $\mathbb{R}^{2}$ is given in polar coordinates by $S=\{(r, \theta): r \geq 0,0 \leq \theta \leq \xi\}$ for some $0<\xi<2 \pi$. Let $\alpha=\left(\theta_{1}+\theta_{2}\right) / \xi$, where $-\pi / 2<\theta_{1}, \theta_{2}<\pi / 2$ are the directions of reflection of $Z$ off each of the two edges of the wedge as measured from the corresponding inward facing normal. We prove that in the case of $1<\alpha<2$, RBM in a wedge is a Dirichlet process. Specifically, its unique Doob-Meyer type decomposition is given by $Z=X+Y$, where $X$ is a two-dimensional Brownian motion and $Y$ is a continuous process of zero energy. Furthermore, we show that for $p>\alpha$, the strong $p$-variation of the sample paths of $Y$ is finite on compact intervals, and, for $0<p \leq \alpha$, the strong $p$-variation of $Y$ is infinite on $[0, T]$ whenever $Z$ has been started from the origin. We also show that on excursion intervals of $Z$ away from the origin, $(Z, Y)$ satisfies the standard Skorokhod problem for $X$. However, on the entire time horizon $(Z, Y)$ does not satisfy the standard Skorokhod problem for $X$, but nevertheless we show that it satisfies the extended Skorkohod problem.
\end{abstract}

Résumé. Le mouvement Brownien réfléchi (RBM) dans un coin est un processus stochastique 2-dimensionnel $Z$ dont l'espace d'états dans $\mathbb{R}^{2}$ est donné en coordonnées polaires par $S=\{(r, \theta): r \geq 0,0 \leq \theta \leq \xi\}$ pour un $0<\xi<2 \pi$. Soit $\alpha=\left(\theta_{1}+\theta_{2}\right) / \xi$, où $-\pi / 2<\theta_{1}, \theta_{2}<\pi / 2$ sont les angles de réflexion de $Z$ sur chacun des côtés du cône, mesurés à partir des normales rentrantes correspondantes. Nous montrons que dans le cas $1<\alpha<2$, le RBM dans un coin est un processus de Dirichlet. Plus précisément, son unique décomposition de Doob-Meyer est donnée par $Z=X+Y$, où $X$ est un mouvement brownien 2-dimensionnel et $Y$ est un processus continu d'énergie zéro. De plus, nous montrons que pour $p>\alpha$, la $p$-variation forte des trajectoires de $Y$ est finie sur les intervalles compacts, et, pour $0<p \leq \alpha$, la $p$-variation forte de $Y$ est infinie sur $[0, T]$ dès que $Z$ est issu de l'origine. Nous montrons aussi que sur les intervalles d'excursions de $Z$ en dehors de l'origine, $(Z, Y)$ satisfait le problème de Skorokhod standard pour $X$. Sur l'intervalle de temps infini, $(Z, Y)$ ne satisfait pas le problème de Skorokhod standard pour $X$, mais satisfait néanmoins le problème de Skorokhod étendu.

MSC: 60J65; 60J27; 60G17; 60G52; 60J55

Keywords: Reflected Brownian motion; Extended Skorokhod problem; Dirichlet process; $p$-variation

\section{Introduction}

Reflected Brownian Motion (RBM) in a wedge is a 2-dimensional stochastic process $Z$ originally defined by Varadhan and Williams [28]. Loosely speaking, this process may be described as follows. Let $X$ be a standard 2-d Brownian motion. Then, $Z$ corresponds to a so-called reflected version of $X$ which is prohibited from exiting a two-dimensional cone or wedge $S$ in $\mathbb{R}^{2}$ with angle $0<\xi<2 \pi$ and vertex at the origin. The process $Y=Z-X$ which is used to constrain $X$ within $S$ is such that its increments may only be in either of the pre-determined directions $v_{1}$ or $v_{2}$ when $Z$ is along the lower and upper boundaries of the wedge, denoted by $\partial S^{1}$ and $\partial S^{2}$, respectively. See Figure 1 for an illustration. For given $X$ finding the pair $(Z, Y)$ is called the Skorokhod problem for $X$. 


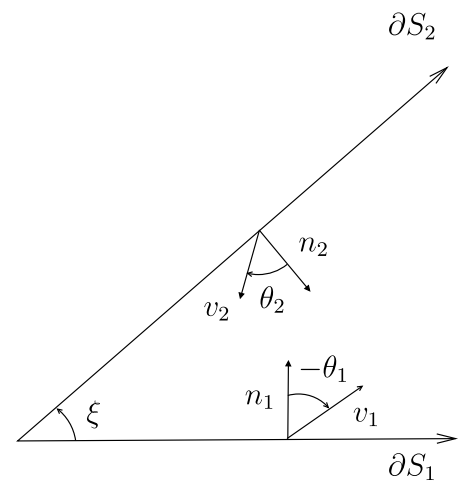

Fig. 1. The state space $S$ of RBM in a wedge.

In the seminal paper [28] Varadhan and Williams defined RBM in a wedge by formulating the submartingale problem, which replaces the quest for a pathwise definition of $Z$ with the search for a family of probability measures on a canonical space equipped with the coordinate-mapping process $Z$. In the results of [28] as well as in those of later papers [29-31] on the subject, the quantity

$$
\alpha=\frac{\theta_{1}+\theta_{2}}{\xi}
$$

plays an important role, where $\theta_{i}$ is the angle of reflection off of the edge $\partial S^{i} \backslash\{0\}, i=1,2$, measured with respect to the inward facing normal vector off each edge, with angles directed toward the origin assumed to be positive. Indeed, in [28] Varadhan and Williams proved that the submartingale problem has a unique solution $\left\{P^{z}, z \in S\right\}$ if and only if $\alpha<2$. However, if one disregards Condition 3 of the submartingale problem which requires that $Z$ spend Lebesguezero amount of time at the vertex, then there exists a unique solution for $\alpha \geq 2$. The tradeoff being that in this case absorption occurs at the vertex. It is also shown in [28] that for every $z \in S$ under $P^{z}$ the coordinate-mapping process $Z$ possesses the strong Markov and the Feller properties.

In [29-31], Williams studied several properties of the solution to the submartingale problem. In particular, in [30] it was proven that $Z$ is a semimartingale under $P^{z}$ if and only if $\alpha<1$ or $\alpha \geq 2$. Or, to state it conversely, $Z$ is not a semi-martingale if and only if $1 \leq \alpha<2$. This naturally raises the following question. What kind of process is $Z$ under $P^{z}$ in the case of $1 \leq \alpha<2$ ? The answer to this question is one of the main contributions of this paper. Recall that any process that may be decomposed as the sum of a local martingale and a process with zero quadratic variation is referred to as a Dirichlet process (see Follmer [18]). Every semimartingale is a Dirichlet process, but the opposite is not necessarily true. Recently it was shown by Kang and Ramanan [19] that if $\alpha=1$, then $Z$ is a Dirichlet process under $P^{z}$. In the present paper we show that $Z$ is a Dirichlet process not only for the single value of $\alpha=1$, but in fact for every value of $\alpha \in(1,2)$. Moreover, we sharpen this result by showing not only that the pushing process $Y$ has zero quadratic variation, but that almost surely on compact intervals of time its strong $p$-variation is finite for $p>\alpha$, and infinite if $0<p \leq \alpha$. Our definition of a Dirichlet process differs slightly from that of Follmer [18], however it is sufficiently strong in order to enable one to apply a change-of-variables formula (Follmer [17]). This then guarantees that a version of Ito's rule can be applied to RBM in a wedge even in cases when $Z$ is not a semimartingale. A useful consequence of the validity of Ito's rule in the case of $\alpha<1$ is that it allows a simple derivation of the Basic Adjoint Relationship (BAR), which is a characterization of the stationary distribution of $Z$. This has been accomplished in several references (see $[12,13]$ and the references therein). The BAR has been successfully used to provide asymptotic estimates, and in some cases provide explicit formula for the density corresponding to the stationary distribution as a sum of exponentials. It is reasonable to expect that using our results and the change-of-variable formula for Dirichlet processes [17] one could proceed in a similar manner to the case of $\alpha<1$ in deriving the BAR. Note also that in our case in order to guarantee the existence of a stationary distribution, one would first need to incorporate an appropriate drift term to the definition of the submartingale problem.

The second problem which we address in this paper is the following. The submartingale problem was clearly formulated with the sample path behavior of $Z$ alluded to in the opening paragraph in mind. In fact, for many values 
of $\alpha$ such behavior has been proven rigorously in [20] by showing that almost surely the pair $(Z, Y)$ pathwise solves an appropriate Skorokhod problem for $X$. However, in the case of $1 \leq \alpha<2$ such a result is impossible since one of the conditions placed on the solution to the Skorokhod problem is that the process $Y$ have bounded variation on compact sets.

In order to address the problem above we use the Extended Skorokhod Problem (ESP) formulated by Ramanan [23]. In a very useful definition, Ramanan [23] relaxed some of the conditions of the Skorokhod problem to allow for pushing processes $Y$ of infinite variation. The heavy traffic diffusion approximation [24] of the queue length process of the Generalized Processor Sharing (GPS) model was given in [23] as one example of a process whose sample paths do not satisfy the conditions of the ordinary Skorokhod problem but do satisfy those of the Extended Skorokhod Problem. In 2 dimensions the GPS model corresponds precisely to RBM in a wedge with a value of $\alpha=1$ and $\xi=\pi / 2$. Burdzy and Toby [5] and also Kang and Ramanan [19] have shown that the ESP has a unique solution for $\alpha=1$ and arbitrary $\xi$. In the present paper we show that if $\left\{P^{z}, z \in S\right\}$ is the solution of the submartingale problem and $\alpha \in(1,2)$, then the pair $(Z, Y)$ solves the ESP for $X$. Thus, we supplement the above examples by providing a rich family of processes for which the SP does not admit a solution but the ESP does.

From an application standpoint, one of the motivations for studying RBM in a wedge is its connection to diffusion approximations for queues in tandem. In particular, a large body of literature exists (see [6] and references therein) rigorously establishing the fact that reflected Brownian in a wedge (and in higher dimensions as well) serves as a limiting approximation to the properly scaled queue length process of a variety of queueing networks as they become fully loaded. This is important because in many cases it turns out that RBM in a wedge is significantly easier to analyze than the underlying queuing network itself. While this connection with queueing theory has not yet been proven in the cases of $1<\alpha<2$ studied in the present paper, we suspect it to be true as well with the coupled processor model $[10,15]$ serving as our underlying example. It is shown by Ramanan and Reiman [24] that such systems may not lead to semimartingales when taking a scaling limit. We expect that the results developed in this paper will form a stepping stone towards the development of a comprehensive theory.

The remainder of this paper is organized as follows. In Section 2, we provide a rigorous problem description followed by our main results. In Section 3, we provide some useful results related to the zero set of RBM in a wedge. Much of the material found in Section 3 is based on the results of Williams [31]. In Section 4, we identify the Brownian motion $X$ which appears in the Doob-Meyer decomposition of $Z$ given by Theorem 2.4. Our proofs of Theorems 2.4, 2.6 and 2.8 may be found in Sections 5, 6 and 7, respectively. Unless otherwise explicitly stated, we assume going forward in the paper that

$$
1<\alpha<2 \text {. }
$$

\section{Main results}

As discussed in the Introduction, reflected Brownian motion (RBM) in a wedge was originally defined by Varadhan and Williams [28] as a 2-dimensional stochastic process $Z$ whose state space in $\mathbb{R}^{2}$ is given in polar coordinates by $S=\{(r, \theta): r \geq 0,0 \leq \theta \leq \xi\}$ for some $0<\xi<2 \pi$. In the interior of $S$, RBM in a wedge behaves as a standard 2-d Brownian, whereas upon hitting the boundary of $S$, it is reflected back into the interior at an angle that depends upon which edge of the boundary has been hit. Let $\partial S^{1}=\{(r, \theta): r \geq 0, \theta=0\}$ and $\partial S^{2}=\{(r, \theta): r \geq 0, \theta=\xi\}$. The angle of reflection off of $\partial S^{1} \backslash\{0\}$ is denoted by $\theta_{1}$, and the angle of reflection off of $\partial S^{2} \backslash\{0\}$ is denoted by $\theta_{2}$. Both of these angles are measured with respect to the inward facing normal off of each edge, with angles directed toward the origin assumed to be positive. The angle of reflection at the vertex of the wedge is discussed later in the paper.

In [28], Varadhan and Williams provided the following rigorous definition of RBM in a wedge as the solution to a submartingale problem. For each $j=1,2$, denote the direction of reflection off of the edge $\partial S^{j} \backslash\{0\}$ by $v_{j}$. Assume moreover that $v_{j}$ is normalized such that $v_{j} \cdot n_{j}=1$, where $n_{j}$ is the inward facing unit normal vector off of the edge $\partial S^{j} \backslash\{0\}$. Let $C_{S}$ denote the space of continuous functions with domain $\mathbb{R}_{+}=[0, \infty)$ and range $S$. For each $t \geq 0$ and $\omega \in C_{S}$, we denote by $Z(t): C_{S} \mapsto S$ the coordinate map $Z(t)(\omega)=Z(t, \omega)=\omega(t)$, and we also define the coordinate mapping process $Z=\{Z(t), t \geq 0\}$. Then, for each $t \geq 0$, we define $\mathcal{M}_{t}=\sigma\{Z(s), 0 \leq s \leq t\}$, and define the process $\mathcal{M}=\sigma\{Z(s), s \geq 0\}$. Next, for each $n \geq 1$ and $F \subset \mathbb{R}^{2}$, denote by $C^{n}(F)$ the set of $n$-times continuously differentiable functions in some domain containing $F$ and let $C_{b}^{n}$ be the set of functions in $C^{n}(F)$ that have bounded partial derivatives up to and including order $n$ on $F$. Finally, define the differential operators $D_{j}=v_{j} \cdot \nabla$ for $j=1,2$, and denote by $\Delta$ the Laplacian operator. 
Definition 2.1 (Varadhan and Williams [28]). A family of probability measures $\left\{P^{z}, z \in S\right\}$ on $\left(C_{S}, \mathcal{M}\right)$ is said to solve the submartingale problem if for each $z \in S$, the following 3 conditions hold,

1. $P^{z}(Z(0)=z)=1$,

2. For each $f \in C_{b}^{2}(S)$, the process

$$
\left\{f(Z(t))-\frac{1}{2} \int_{0}^{t} \Delta f(Z(s)) d s, t \geq 0\right\}
$$

is a submartingale on $\left(C_{S}, \mathcal{M}, \mathcal{M}_{t}, P^{z}\right)$ whenever $f$ is constant in a neighborhood of the origin and satisfies $D_{i} f \geq 0$ on $\partial S^{i}$ for $i=1,2$,

3.

$$
E^{z}\left[\int_{0}^{\infty} 1\{Z(t)=0\} d t\right]=0
$$

Now recall the definition of $\alpha=\left(\theta_{1}+\theta_{2}\right) / \xi$ from (1) in the Introduction. In [28], it was shown that if $\alpha<2$, then there exists a unique solution $\left\{P^{z}, z \in S\right\}$ to the submartingale problem. Moreover [28], the family $\left\{P^{z}, z \in S\right\}$ possess the strong Markov property. If $\alpha \geq 2$, then there is no solution [28] to the submartingale problem but there is a unique family of probability measures $\left\{P^{z}, z \in S\right\}$ satisfying Conditions 1 and 2 of Definition 2.1. Probabilistically speaking, the process $Z$ is absorbed upon reaching the origin when $\alpha \geq 2$ and hence may spend a positive amount of Lebesgue time there so that Condition 3 no longer holds. In this case, we may continue to refer to $\left\{P^{z}, z \in S\right\}$ as the solution to the submartingale problem, with the understanding that only Conditions 1 and 2 of Definition 2.1 are satisfied.

Now, as in Williams [29], let $\mathcal{B}_{S}$ denote the Borel $\sigma$-algebra on $S$ and for each probability measure $\mu$ on $\left(S, \mathcal{B}_{S}\right)$, define the measure $P^{\mu}$ on $\left(C_{S}, \mathcal{M}\right)$ by setting

$$
P^{\mu}(A)=\int_{S} \mu(d z) P^{z}(A), \quad A \in \mathcal{M}
$$

We then denote by $\left(C_{S}, \mathcal{M}^{\mu}, P^{\mu}\right)$ the completion of $\left(C_{S}, \mathcal{M}, P^{\mu}\right)$ and for each $t \geq 0$, we let $\mathcal{M}_{t}^{\mu}$ be the augmentation of $\mathcal{M}_{t}$ with respect to $\left(C_{S}, \mathcal{M}^{\mu}, P^{\mu}\right)$. If $\mu=\delta_{\{z\}}$ for some $z \in S$ (the Dirac measure centered at $z$ ), then we shall write $\mathcal{M}^{z}$ and $\mathcal{M}_{t}^{z}$ instead of $\mathcal{M}^{\mu}$ and $\mathcal{M}_{t}^{\mu}$. Next, define $\mathcal{F}=\bigcap_{\mu} \mathcal{M}^{\mu}$, where the intersection is taken over all $\mu$ which are probability measures on $\left(S, \mathcal{B}_{S}\right)$, and similarly set $\mathcal{F}_{t}=\bigcap_{\mu} M_{t}^{\mu}$. It then follows [29] that the filtration $\mathcal{F}_{t}$ is right-continuous and, moreover, the process $\mathcal{Z}=\left(C_{S}, \mathcal{F}, \mathcal{F}_{t}, Z(t), \theta_{t}, P^{z}\right)$ is a Hunt process with state space $\left(S, \mathcal{B}_{S}\right)$, where $\theta_{t}$ is the usual shift operator.

\subsection{Dirichlet process result}

As mentioned in the Introduction, in [30] Williams proved that for each $z \in S, Z$ is a semimartingale on $\left(C_{S}, \mathcal{F}, \mathcal{F}_{t}, P^{z}\right)$ if and only if $\alpha<1$ or $\alpha \geq 2$. More recently, Kang and Ramanan [19] have proven that in the boundary case of $\alpha=1, Z$ is a Dirichlet process (see Definition 2.3 below) on $\left(C_{S}, \mathcal{F}, \mathcal{F}_{t}, P^{z}\right)$ for each $z \in S$. The proofs of the results in [19] rest upon several bounds. One of the most important of these bounds is Assumption 2 of [19] which requires that for some $p>1$ and $q \geq 2$ the conditional expectation of the $p$ th power of the oscillation of the pushing process is dominated by the conditional expectation of the $q$ th power of the oscillation of the Brownian motion over any finite interval $[s, t]$. In the case of RBM in a wedge, this has only been shown to hold in the special case of $\alpha=1$ (see Ramanan [23]). As a result, the approach of [19] cannot be easily extended to the remaining cases of $1<\alpha<2$. On the other hand, there are many discrete models, such as the coupled processor model of Fayolle and Isanogorodski [15], for which RBM in a wedge with $1<\alpha<2$ is conjectured to arise as the natural scaling limit (see also the dissertation of Foddy [16] for a discussion of the case $\alpha<1$ ). In the present paper, using newly developed techniques, we prove in Theorem 2.4 below that in the cases of $1<\alpha<2, Z$ is in fact a Dirichlet process on $\left(C_{S}, \mathcal{F}, \mathcal{F}_{t}, P^{z}\right)$ for each $z \in S$. This implies, for instance, that one may apply a change-of-variables formula to $Z$ in order to obtain further results. 
For each $E \subseteq \mathbb{R}_{+}$, let

$$
\pi(E)=\left\{\pi=\left\{t_{i}, i=0, \ldots, n\right\} \subseteq E: n \in \mathbb{N}_{0}, t_{i-1} \leq t_{i}, i=1, \ldots, n\right\}
$$

denote the set of all partitions of $E$, and define the mesh of a partition $\pi \in \pi(E)$ by setting

$$
\|\pi\|=\max \left\{\left|t_{i}-t_{i-1}\right|: i=1, \ldots, n\right\} .
$$

We then recall the definition of a continuous process with zero energy (see, for instance, Definition 3.2 of Kang and Ramanan [19]).

Definition 2.2. Let $z \in S$. A 2-dimensional continuous process $Y$ defined on $\left(C_{S}, \mathcal{F}, \mathcal{F}_{t}, P^{z}\right)$ is said to be of zero energy if for each $T>0$,

$$
\sum_{t_{i} \in \pi^{n}}\left\|Y\left(t_{i}\right)-Y\left(t_{i-1}\right)\right\|^{2} \stackrel{P}{\rightarrow} 0 \quad \text { as } n \rightarrow \infty,
$$

for any sequence $\left\{\pi^{n}, n \geq 1\right\}$ of partitions of $[0, T]$ with $\left\|\pi^{n}\right\| \rightarrow 0$ as $n \rightarrow \infty$.

We now define a Dirichlet process as follows (see Definition 2.4 of Coquet et al. [11] or Definition 3.3 of [19]).

Definition 2.3. Let $z \in S$. The stochastic process $Z$ is said to be a Dirichlet process on $\left(C_{S}, \mathcal{F}, \mathcal{F}_{t}, P^{z}\right)$ if we may write

$$
Z=X+Y,
$$

where $X$ is an $\mathcal{F}_{t}$-adapted local martingale and $Y$ is a continuous, $\mathcal{F}_{t}$-adapted zero energy process with $Y(0)=0$.

The above definition of a Dirichlet process differs slightly from that of Föllmer [18]. However, it is sufficiently strong in order to enable one to obtain formulas for performing certain operations on $Z$, such as a change-of-variables (see Föllmer [17]). This can be useful in further analysis, for instance in analyzing the transient behavior of the process. Note also that if $Z$ is a semi-martingale, then it is also a Dirichlet process as defined above. However, the converse is not necessarily true. It may also be shown that the Doob-Meyer type decomposition of $Z$ given by (2) is unique (see Remark 3.4 of [19]).

Theorem 2.4. Suppose that $1<\alpha<2$. Then, $Z$ has the decomposition

$$
Z=X+Y
$$

where $(X, Y)$ is a pair of processes on $\left(C_{S}, \mathcal{F}, \mathcal{F}_{t}\right)$ and for each $z \in S$ the following hold:

- $X$ is a standard 2-dimensional Brownian motion under $P^{z}$ such that $X(0)=z, P^{z}$-a.s.;

- $Y$ is a process of zero energy under $P^{z}$.

In particular, for each $z \in S$, the process $Z$ is a Dirichlet process on $\left(C_{S}, \mathcal{F}, \mathcal{F}_{t}, P^{z}\right)$.

The Brownian motion $X$ appearing in Theorem 2.4 is the same as the one established in Kang and Ramanan [20]. However, we have a slightly different definition in order to facilitate the proof of Lemma 4.11. The exact difference will be pointed out at the beginning of Section 4.1.

Our construction of the Brownian motion $X$ appearing in Theorem 2.4 is similar to the corresponding construction found in Kang and Ramanan [20]. However, here we also prove that $(Z, Y)$ solves a corresponding Skorokhod problem for $X$ on excursion intervals of $Z$ away from the origin. This result is then used together with the local behavior of $Z$ at the origin in order to piece together the process $Y$ on a excursion-by-excursion interval basis. In particular, we show that for each $p>\alpha, Y$ is a composition of a locally Hölder continuous function with exponent $1 / p$, and a nondecreasing continuous function. Using the fact that $1<\alpha<2$, it then follows in a straightforward manner that $Y$ is of 
zero energy. We also emphasize that the pair of processes $(X, Y)$ appearing in Theorem 2.4 do not depend on $z \in S$. That is, there exists a single pair of processes $(X, Y)$ on $\left(C_{S}, \mathcal{F}, \mathcal{F}_{t}\right)$ such that the statement of the above theorem holds for each $z \in S$.

\subsection{Roughness of the paths of $Y$ result}

Theorem 2.4 implies that in the case of $1<\alpha<2$, the paths of $Y$ are of zero energy on $\left(C_{S}, \mathcal{F}, \mathcal{F}_{t}, P^{z}\right)$ for each $z \in S$. We now turn our attention to providing a refinement of this result using the concept of strong $p$-variation.

Definition 2.5. Let $E \subset \mathbb{R}_{+}$and $p>0$. The strong $p$-variation of a function $f: \mathbb{R}_{+} \mapsto \mathbb{R}^{n}$ on $E$ is defined by

$$
V_{p}(f, E)=\sup \left\{\sum_{t_{i} \in \pi}\left\|f\left(t_{i}\right)-f\left(t_{i-1}\right)\right\|^{p}: \pi \in \pi(E)\right\} .
$$

Theorem 2.6. Suppose that $1<\alpha<2$. Then, for each $p>\alpha$ and $z \in S$,

$$
P^{z}\left(V_{p}(Y,[0, T])<+\infty\right)=1, \quad T \geq 0 .
$$

Furthermore, for each $0<p \leq \alpha$,

$$
P^{0}\left(V_{p}(Y,[0, T])<+\infty\right)=0, \quad T \geq 0 .
$$

Our proof of Theorem 2.6 has two parts. In order to prove the identity (4), we rely upon the relationship between functions of finite $p$-variation and Hölder continuous functions with exponent $1 / p$. In particular, since as alluded to after the statement of Theorem 2.4 above, for each $p>\alpha, Y$ is a composition of a locally Hölder continuous function with exponent $1 / p$, and a non-decreasing continuous function, it is straightforward to show that $Y$ also has finite $p$-variation on compact intervals. Our proof of the identity (5) proceeds by first showing that on a specific subset of the zero set of $Z$, the process $Y$ is a stable process of index $\alpha$ if $Z$ is started from the origin. It then follows from available results in the literature (see, for instance, Bretagnolle [3]) that $Y$ has infinite $p$-variation for $0<p \leq \alpha$.

\subsection{Extended Skorokhod map result}

We now provide an additional connection between the pair of processes $(X, Y)$ appearing in Theorem 2.4 and the process $Z$ itself. Let $D\left(\mathbb{R}_{+}, \mathbb{R}^{2}\right)$ denote the space of $\mathbb{R}^{2}$-valued functions, with domain $\mathbb{R}_{+}$, that are right-continuous with left limits. Also, let $D_{S}\left(\mathbb{R}_{+}, \mathbb{R}^{2}\right)$ be the set of $f \in D\left(\mathbb{R}_{+}, \mathbb{R}^{2}\right)$ such that $f(0) \in S$. Next, let $d(\cdot)$ be a set-valued mapping defined on $S$ such that $d(z)$ is a closed convex cone in $\mathbb{R}^{2}$ for every $z \in S$. In particular, we define

$$
d(z)= \begin{cases}\left\{a v_{1}, a \geq 0\right\}, & \text { for } z \in \partial S^{1} \backslash\{0\}, \\ \left\{a v_{2}, a \geq 0\right\}, & \text { for } z \in \partial S^{2} \backslash\{0\}, \\ V, & \text { for } z=0, \\ \{0\}, & \text { for } z \in \operatorname{int}(S),\end{cases}
$$

where the selection of the closed convex cone $V \subset \mathbb{R}^{2}$ will be discussed in a moment. For a set $A \subseteq \mathbb{R}^{2}$, let $\overline{\operatorname{co}}(A)$ denote the closure of the convex hull of $A$. We then recall from Ramanan [23] the definition of the extended Skorokhod problem (ESP).

Definition 2.7. The pair of processes $(\phi, \eta) \in D_{S}\left(\mathbb{R}_{+}, \mathbb{R}^{2}\right) \times D\left(\mathbb{R}_{+}, \mathbb{R}^{2}\right)$ solve the $\operatorname{ESP}(S, d(\cdot))$ for $\psi \in D_{S}\left(\mathbb{R}_{+}, \mathbb{R}^{2}\right)$ if $\phi(0)=\psi(0)$, and if for all $t \in \mathbb{R}_{+}$, the following properties hold,

1. $\phi(t)=\psi(t)+\eta(t)$,

2. $\phi(t) \in S$, 
3. For every $s \in[0, t]$,

$$
\eta(t)-\eta(s) \in \overline{\mathrm{co}}\left[\bigcup_{u \in(s, t]} d(\phi(u))\right],
$$

4. $\eta(t)-\eta(t-) \in \overline{\operatorname{co}}[d(\phi(t))]$.

Theorem 2.8. Suppose that $1<\alpha<2$. Then, for each $z \in S$, the $\operatorname{ESP}(S, d(\cdot))$ for the Brownian motion $X$ on $\left(C_{S}, \mathcal{F}, \mathcal{F}_{t}, P^{z}\right)$ has a solution $P^{z}$-a.s. if and only if

$$
\overline{\mathrm{co}}\left(V \cup\left\{a v_{1}, a \geq 0\right\} \cup\left\{a v_{2}, a \geq 0\right\}\right)=\mathbb{R}^{2} .
$$

In this case, $(Z, Y)$ solves the $\operatorname{ESP}(S, d(\cdot))$ for $X$.

By trivially setting $V=\mathbb{R}^{2}$, it follows that one may always find a $V$ such that (7) holds. However, using the fact that $1<\alpha<2$, it is straightforward to verify that the smaller set $V=\left\{a v_{0}, a \geq 0\right\}$ for any $v_{0}$ in the interior of $S$ satisfies (7) as well. Note also that we stop short of claiming in Theorem 2.8 that $(Z, Y)$ is the unique solution to the $\operatorname{ESP}(S, d(\cdot))$ for $X$.

\section{Results related to the zero set of $Z$}

The following results are helpful as we proceed throughout the paper. Let $\Lambda=\left\{t \in \mathbb{R}_{+}: Z(t)=0\right\}$ denote the zero set of $Z$ and note that since $Z$ is a continuous process for every $\omega \in C_{S}$, it follows that $\Lambda$ is a closed set for every $\omega \in C_{S}$. Next, let $z \in S$ and recall (see Definition 4.2.9 of [21]) that the origin is said to be regular for $Z$ under $P^{z}$ if $P^{z}\left(\sigma_{\overline{0}}=0\right)=1$, where $\sigma_{\overline{0}}=\inf \{t>0: Z(t) \neq 0\}$. Then, since by Lemma 2.2 of Williams [31] the origin is regular for $Z$ under $P^{z}$ for each $z \in S$, using standard arguments (see Theorem 2.9.6 of Karatzas and Shreve [21]) it may be shown that for each $z \in S$ the set $\Lambda$ contains no isolated points $P^{z}$-a.s. Next, let $\Lambda^{C}=\left\{t \in \mathbb{R}_{+}: Z(t) \neq 0\right\}$ denote the complement of $\Lambda$ in $\mathbb{R}_{+}$. It then follows that $\Lambda^{C}$ is an open set in $\mathbb{R}_{+}$, and in fact it may be written as

$$
\Lambda^{C}=\left[0, \tau_{0}\right) \cup\left(\bigcup_{i=1}^{\infty}\left(G_{i}, D_{i}\right)\right),
$$

where $\tau_{0}$ is the first hitting time of the origin by $Z$, i.e.,

$$
\tau_{0}=\inf \{t>0: Z(t)=0\} .
$$

Note that $\tau_{0}=0, P^{0}$-a.s., in which case we interpret $[0,0)$ as the empty set. For each $i \geq 1$, we refer to $\left(G_{i}, D_{i}\right)$ as an excursion interval of $Z$ away from the vertex and refer to the paths of $Z$ over such intervals as excursions from the vertex. It may easily be shown that the sequence of random times $\left\{\left(G_{i}, D_{i}\right), i \geq 1\right\}$ can be selected such that $\left(G_{i}, D_{i}\right): C_{S} \mapsto \mathbb{R}_{+}^{2}$ is a measurable function on $\left(C_{S}, \mathcal{F}\right)$ for each $i \geq 1$. We assume for the remainder of the paper that such a selection has been made.

Closely related to the zero set $\Lambda$ is the concept of the local time of $Z$ at the origin. Since the origin is a regular point for $Z$, it follows by the results of Blumenthal and Getoor [2] that there exists a continuous, non-decreasing, perfect, adapted functional $L$ with support $\{0\}$. We refer to this process as the local time of $Z$ at the origin. Moreover, the results of [2] also guarantee that $L$ is uniquely determined up to a multiplicative constant. As in [31], we assume for the remainder of the paper that the multiplicative constant is chosen such that

$$
E^{0}\left[\int_{0}^{\infty} e^{-t} d L(t)\right]=1 .
$$

Now note that since $1<\alpha<2$, it is straightforward to show using Theorem 2.2 of Varadhan and Williams [28], together with the strong Markov property [28] of $Z$, that $L(t) \rightarrow \infty$ as $t \rightarrow \infty, P^{z}$-a.s. for each $z \in \mathrm{S}$. Hence, we may 
$P^{z}$-a.s. define the inverse local time process

$$
L^{-1}(a)=\inf \{t \geq 0: L(t)>a\}, \quad a \geq 0,
$$

and we set $L^{-1}=\left\{L^{-1}(a), a \geq 0\right\}$. By Corollary 2.7 of Williams [31], under $P^{0}, L^{-1}$ is a stable subordinator of index $\alpha / 2$. In particular, $\ln E^{0}\left[e^{-\lambda L^{-1}(a)}\right]=-a \lambda^{\alpha / 2}$ for $a, \lambda \geq 0$. Hence, under $P^{0}, L^{-1}$ is a strictly increasing, right-continuous, pure jump process (see Bertoin [1]), and so we have that $P^{0}$-a.s.,

$$
L^{-1}(a)=\sum_{0 \leq \kappa \leq a} \Delta L^{-1}(\kappa), \quad a \geq 0
$$

where

$$
\Delta L^{-1}(\kappa)=L^{-1}(\kappa)-L^{-1}(\kappa-), \quad \kappa \geq 0,
$$

with the convention that $L^{-1}(0-)=0$. In particular, whenever $\Delta L^{-1}(\kappa) \neq 0$, then it is straightforward to show that $\Delta L^{-1}(\kappa)=D_{i}-G_{i}$ for the unique value of $i \geq 1$ such that $L\left(G_{i}\right)=L\left(D_{i}\right)=\kappa$. Thus, we also have that $P^{0}$-a.s.,

$$
L^{-1}(a)=\sum_{i: L\left(G_{i}\right) \leq a}\left(D_{i}-G_{i}\right), \quad a \geq 0 .
$$

We note that using arguments similar to the proof of Corollary 5.11 (page 411) of Çinlar [8], it may be shown that $P^{0}$-a.s., $L^{-1}\left(\mathbb{R}_{+}\right) \subset \Lambda$ with

$$
\Lambda \backslash L^{-1}\left(\mathbb{R}_{+}\right)=\bigcup_{i=1}^{\infty}\left\{G_{i}\right\} .
$$

Finally, a note on notation. It is customary to write $L(t)$ instead of $L(t, \omega)=L(t, Z(\cdot, \omega))$, and we shall do so. However, in Section 6 we need to write down the local time calculated for an element of $C_{s}$ other than $\omega=Z(\cdot, \omega)$. For example, for the additivity property, we need to write down the local time at time $t-s$ applied to $Z(s+\cdot, \omega)$ for some $0 \leq s \leq t$. In order to strike a compromise between clarity and brevity, we shall write $L(t)$ instead of $L(t, \omega)=L(t, Z(\cdot, \omega))$. However, for $u, s \geq 0$ we shall write $L(u, Z(s+\cdot))$ instead of $L(u, Z(s+\cdot, \omega))$. For example, we write the additivity property as

$$
L(t)=L(s)+L(t-s, Z(s+\cdot)),
$$

$P^{z}$-a.s., for all $z \in S$, and $0 \leq s \leq t$. Note that the exceptional set on which (13) does not hold does not depend on $s$ and $t$ since $L$ is assumed to be perfect.

\section{Identification of $X$ and the standard Skorokhod problem}

In this section, we prove the existence of a 2-dimensional process $X$ on $\left(C_{s}, \mathcal{F}, \mathcal{F}_{t}\right)$ which is a standard 2-dimensional Brownian motion started from $z$ on $\left(C_{S}, \mathcal{F}, \mathcal{F}_{t}, P^{z}\right)$ for each $z \in S$. Moreover, letting $Y=Z-X$, we show that over each excursion interval $\left[G_{i}, D_{i}\right]$ for $i \geq 1$ (and if $z \neq 0$, then also over the interval from zero to the first time that $Z$ hits the origin), the pair of processes $(Z, Y)$ solves the standard Skorokhod problem for $X$ given by Definition 4.1 below. In Section 5, it is proven that the process $Y$ is of zero energy on $\left(C_{S}, \mathcal{F}, \mathcal{F}_{t}, P^{z}\right)$ for each $z \in S$, and hence $(X, Y)$ is the desired Doob-Meyer type decomposition of $Z$ given by Theorem 2.4. We also note that our construction of the process $X$ is similar to that of Kang and Ramanan [20], but differs in an important way in order to allow us to prove our results related the Skorokhod problem.

We now recall the definition of the standard Skorokhod problem (SP) as given in Ramanan [23]. Recall first from Section 2.3 the definition of $D\left(\mathbb{R}_{+}, \mathbb{R}^{2}\right)$ as the space of $\mathbb{R}^{2}$-valued functions, with domain $\mathbb{R}_{+}$, that are rightcontinuous with left limits, and the definition of $D_{S}\left(\mathbb{R}_{+}, \mathbb{R}^{2}\right)$ as the set of $f \in D\left(\mathbb{R}_{+}, \mathbb{R}^{2}\right)$ such that $f(0) \in S$. Also, let $\mathcal{B} V\left(\mathbb{R}_{+}, \mathbb{R}^{2}\right) \subset D\left(\mathbb{R}_{+}, \mathbb{R}^{2}\right)$ denote the subset of functions in $D\left(\mathbb{R}_{+}, \mathbb{R}^{2}\right)$ which have finite variation on each bounded 
interval. For each $f \in \mathcal{B} V\left(\mathbb{R}_{+}, \mathbb{R}^{2}\right)$ and $t \geq 0$, we denote the variation of $f$ on $[0, t]$ by $\bar{f}(t)$, which is a shorthand notation for $V_{1}(f,[0, t])$. Next, recall from (6) of Section 2.3, the definition of the set-valued mapping $d(\cdot)$ defined on $S$, and, for each $z \in S$, let $d^{1}(z)$ denote the intersection of $d(z)$ with the unit sphere $S_{1}(0)$ in $\mathbb{R}^{2}$ which is centered at the origin. We then recall from Ramanan [23] the definition of the standard Skorokhod problem (SP).

Definition 4.1. The pair of processes $(\phi, \eta) \in D_{S}\left(\mathbb{R}_{+}, \mathbb{R}^{2}\right) \times D\left(\mathbb{R}_{+}, \mathbb{R}^{2}\right)$ solves the $\mathrm{SP}(S, d(\cdot))$ for $\psi \in D_{S}\left(\mathbb{R}_{+}, \mathbb{R}^{2}\right)$ if $\phi(0)=\psi(0)$, and if for all $t \in \mathbb{R}_{+}$, the following properties hold,

1. $\phi(t)=\psi(t)+\eta(t)$,

2. $\phi(t) \in S$,

3. $\eta \in \mathcal{B} V\left(\mathbb{R}_{+}, \mathbb{R}^{2}\right)$,

4. $\bar{\eta}(t)=\int_{[0, t]} 1\{\phi(s) \in \partial S\} d \bar{\eta}(s)$,

5. There exists a measurable function $\gamma: \mathbb{R}_{+} \mapsto S_{1}(0)$ such that $d \bar{\eta}$-almost everywhere we have that $\gamma(t) \in d^{1}(\phi(t))$ and

$$
\eta(t)=\int_{0}^{t} \gamma(s) d \bar{\eta}(s)
$$

Our main result of this section is the following.

Theorem 4.2. Suppose that $1<\alpha<2$.

1. There exists a 2-dimensional process $X$ on $\left(C_{S}, \mathcal{F}, \mathcal{F}_{t}\right)$ such that $X$ is a standard 2-dimensional Brownian motion started from $z$ on $\left(C_{S}, \mathcal{F}, \mathcal{F}_{t}, P^{z}\right)$ for each $z \in S$.

2. Let $X$ be as above, $Y=Z-X$, and let $V$ in (6) be an arbitrary, non-empty closed convex cone in $\mathbb{R}^{2}$. Then, for each $z \in S, P^{z}$-a.s. the pair $\left(Z\left(\left(G_{i}+\cdot\right) \wedge D_{i}\right), Y\left(\left(G_{i}+\cdot\right) \wedge D_{i}\right)-Y\left(G_{i}\right)\right)$ solves the $S P(S, d(\cdot))$ for $X\left(\left(G_{i}+\right.\right.$ $\left.\cdot) \wedge D_{i}\right)+Y\left(G_{i}\right)$ for each $i \geq 1$. In addition, for each $z \in S \backslash\{0\}, P^{z}$-a.s. the pair $\left(Z\left(\cdot \wedge \tau_{0}\right), Y\left(\cdot \wedge \tau_{0}\right)\right)$ solves the $S P(S, d(\cdot))$ for $X\left(\cdot \wedge \tau_{0}\right)$.

The two parts of Theorem 4.2 are proven respectively in Sections 4.1 and 4.2 below. Also note that $d(0)=V$ in the definition of $d(\cdot)$, and the selection of $V$ turns out to be important in Theorem 2.8. However, since $Z$ does not reach the origin on $\left(G_{i}, D_{i}\right)$, or on $\left[0, \tau_{0}\right)$ if $z \neq 0$, the selection of $V$ in the above theorem is irrelevant.

\subsection{Proof of Part 1 of Theorem 4.2}

For each $\delta>0$, let $S_{\delta} \subset S$ be the closed set defined in polar coordinates by $S_{\delta}=S+\delta e^{i \xi / 2}$. Next, set $\tau_{0}^{\delta}=0$, and, for each $k \geq 1$, recursively define

$$
\sigma_{k}^{\delta}=\inf \left\{t \geq \tau_{k-1}^{\delta}: Z(t) \in S_{2 \delta}\right\} \quad \text { and } \quad \tau_{k}^{\delta}=\inf \left\{t \geq \sigma_{k}^{\delta}: Z(t) \in \partial S_{\delta}\right\} .
$$

In the definition of $\sigma_{k}^{\delta}$ we have $S_{2 \delta}$ instead of $\partial S_{2 \delta}$ because in the case when $Z(0)$ lies in the interior of $S_{2 \delta}$ we want $\sigma_{1}^{\delta}$ to be defined zero. The time intervals $\left[\sigma_{k}^{\delta}, \tau_{k}^{\delta}\right]$ define the successive excursions of $Z$ from the boundary of $S_{2 \delta}$ to the boundary of $S_{\delta}$, except that for $k=1$ when $Z(0)$ is in the interior of $S_{2 \delta}$ then $\left[\sigma_{1}^{\delta}, \tau_{1}^{\delta}\right]$ is the excursion interval from $Z(0)$ to the boundary of $S_{\delta}$. See Figure 2.

Since $Z(\cdot, \omega)$ is continuous for every $\omega \in C_{S}$, we may equivalently set $\tau_{k}^{\delta}=\inf \left\{t \geq \sigma_{k}^{\delta}: Z(t) \in S_{\delta}^{c} \cap S^{o}\right\}$, where $S_{\delta}^{c}$ denotes the complement of $S_{\delta}$ in $S$, and $S^{o}$ is the interior of $S$. It is immediate by Proposition 2.1.5 of Ethier and Kurtz [14] that $\sigma_{k}^{\delta}$ and $\tau_{k}^{\delta}$ are, for each $k \geq 1$, stopping times relative to the right-continuous filtration $\left\{\mathcal{F}_{t}, t \geq 0\right\}$.

Now, for each $k \geq 1$, let $W_{(k)}^{\delta}$ be the process defined by setting

$$
W_{(k)}^{\delta}(t)=Z\left(t \wedge \tau_{k}^{\delta}\right)-Z\left(t \wedge \sigma_{k}^{\delta}\right), \quad t \geq 0,
$$

and then define the process $W^{\delta}$ by setting

$$
W^{\delta}(t)=\sum_{k=1}^{\infty} W_{(k)}^{\delta}(t), \quad t \geq 0 .
$$




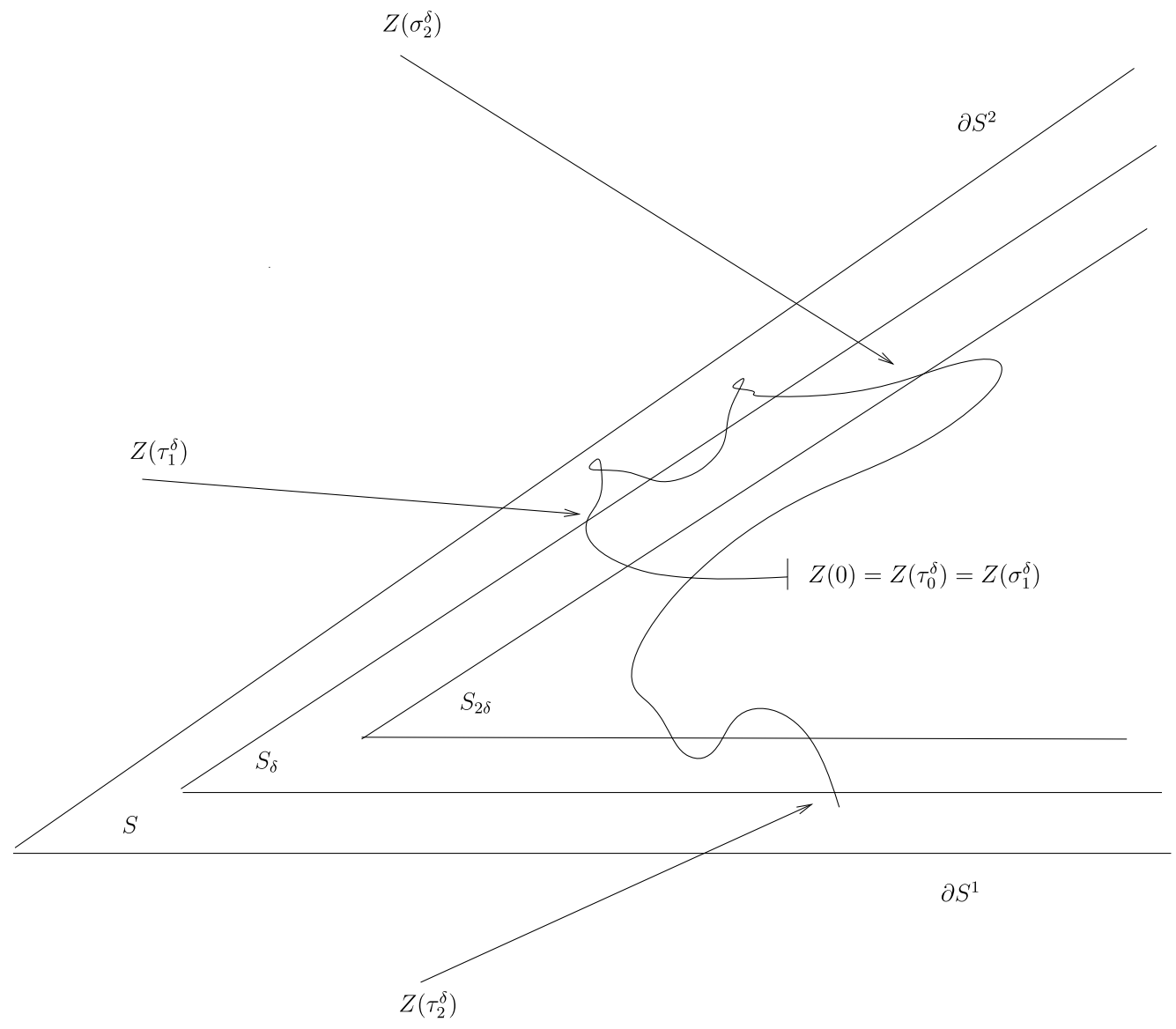

Fig. 2. Basic notations.

This process is not exactly the same as the corresponding process in Kang and Ramanan [20] defined under (5.7) there (it is denoted by $W^{m}$ in that paper). It can be shown though that in the limit (as $\delta \rightarrow 0$ here and $m \rightarrow \infty$ in Kang and Ramanan [20]) these two definitions lead to the same Brownian motion. However, the present definition is more convenient to prove a pathwise functional relationship between the solution of the submartingale problem and the Brownian motion, that will be accomplished in Lemma 4.11.

Recall next from Section 2 the definition of the augmented sigma fields $\mathcal{M}^{z}$ and $\mathcal{M}_{t}^{z}$ for $z \in S$ and $t \geq 0$. For each $z \in S$, let $\Upsilon_{2}^{c, z}$ denote the space of continuous, square-integrable martingales $M$ with time horizon $\mathbb{R}_{+}$on $\left(C_{S}, \mathcal{M}^{z}, \mathcal{M}_{t}^{z}, P^{z}\right)$, and such that $M(0)=0$. It is well-known (see for instance Proposition 1.5.23 of Karatzas and Shreve [21]) that the space $\Upsilon_{2}^{c, z}$ is complete under the norm

$$
\|M\|_{z}=\sum_{n=1}^{\infty} \frac{1}{2^{n}}\left(\sqrt{E^{z\left[M^{2}(n)\right]}} \wedge 1\right), \quad M \in \Upsilon_{2}^{c, z} .
$$

Now, for each $\delta>0$ and $k \geq 1$ and $i=1,2$, denote the $i$ th component process of $W_{(k)}^{\delta}$ by $W_{(k), i}^{\delta}$. We begin our proof of Part 1 of Theorem 4.2 by recalling a result from Kang and Ramanan [20].

Lemma 4.3. For each $\delta>0, k \geq 1, i=1,2$, and $z \in S$, the process $W_{(k), i}^{\delta} \in \Upsilon_{2}^{c, z}$ with quadratic variation processes

$$
\left\langle W_{(k), i}^{\delta}\right\rangle_{t}=\left(t \wedge \tau_{k}^{\delta}\right)-\left(t \wedge \sigma_{k}^{\delta}\right), \quad t \geq 0,
$$

for $i=1,2$, and $\left\langle W_{(k), 1}^{\delta}, W_{(k), 2}^{\delta}\right\rangle_{t}=0, t \geq 0$. 
Proof. This follows from Lemma 5.1 in [20].

Lemma 4.4. For each $\delta>0$ and $k, \ell \geq 1$ with $k \neq \ell$, we have under $P^{z}$ for every $z \in S$ that

$$
\left\langle W_{(k), i}^{\delta}, W_{(\ell), j}^{\delta}\right\rangle_{t}=0, \quad t \geq 0,
$$

for $i, j=1,2$.

Proof. This follows immediately from (14).

Lemma 4.5. For each $z \in S, \delta>0$ and $i=1,2$, we have that $W_{i}^{\delta} \in \Upsilon_{2}^{c, z}$ with quadratic and cross variations

$$
\left\langle W_{i}^{\delta}\right\rangle_{t}=\sum_{k=1}^{\infty}\left(\left(t \wedge \tau_{k}^{\delta}\right)-\left(t \wedge \sigma_{k}^{\delta}\right)\right), \quad t \geq 0,
$$

and $\left\langle W_{1}^{\delta}, X_{2}^{\delta}\right\rangle_{t}=0, t \geq 0$.

Proof. All these statements follow immediately from Lemmas 4.3 and 4.4.

Let $\Gamma$ be the set of sequences $\{a(n), n \geq 1\}$ such that $a(n) \downarrow 0$ as $n \rightarrow \infty$ and $2 a(n+1)<a(n)$ for every $n \geq 1$. The set $\Gamma$ will be useful for the following reason. Suppose that $\{a(n), n \geq 1\} \in \Gamma$ and let $n>m \geq 1$ arbitrary. Then for every $k \geq 1$ there exists an integer $l \geq 1$ such that $\left[\sigma_{k}^{a(m)}, \tau_{k}^{a(m)}\right] \subset\left[\sigma_{l}^{a(n)}, \tau_{l}^{a(n)}\right]$. In other words, every excursion from $\partial S_{2 a(m)}$ to $\partial S_{a(m)}$ is included in an excursion from $\partial S_{2 a(n)}$ to $\partial S_{a(n)}$.

Lemma 4.6. For every sequence $\{\delta(n), n \geq 1\} \in \Gamma$ we have that $\left\{W_{i}^{\delta(n)}, n \geq 1\right\}$ is Cauchy in $\Upsilon_{2}^{c, z}$ for every $z \in S$ and $i=1,2$.

Proof. Let $\{\delta(n), n \geq 1\} \in \Gamma$. Clearly it is sufficient to prove that $\left\{W_{i}^{\delta(n)}(T), n \geq 1\right\}$ is Cauchy in $L^{2}\left(C_{S}, \mathcal{F}, P^{z}\right)$ for each $T \geq 0$. Suppose that for each $n>m \geq 1$ we show that

$$
E^{z}\left[\left(W_{i}^{\delta(n)}(T)-W_{i}^{\delta(m)}(T)\right)^{2}\right] \leq E^{z} \int_{0}^{T} 1\left\{Z(t) \in S \backslash S_{2 \delta(m)}\right\} d t .
$$

Then by the Dominated Convergence Theorem and Lemma 4.2 of Williams [29] we have that

$$
\lim _{m \rightarrow \infty} E^{z}\left[\int_{0}^{T} 1\left\{Z(t) \in S \backslash S_{2 \delta(m)}\right\} d t\right]=E^{z}\left[\int_{0}^{T} 1\{Z(t) \in \partial S\} d t\right]=0,
$$

and so $\left\{W_{i}^{\delta(n)}(T), n \geq 1\right\}$ is Cauchy in $L^{2}\left(C_{S}, \mathcal{F}, P^{z}\right)$ as desired.

Hence we need to show only (17), and that is what we shall do in the rest of this proof. Now write

$$
\begin{aligned}
E^{z}\left[\left(W_{i}^{\delta(n)}(T)-W_{i}^{\delta(m)}(T)\right)^{2}\right]= & E^{z}\left[\left\langle W_{i}^{\delta(n)}-W_{i}^{\delta(m)}\right\rangle_{T}\right] \\
= & E^{z}\left[\sum_{k=1}^{\infty}\left[\left\langle W_{i}^{\delta(n)}-W_{i}^{\delta(m)}\right\rangle_{\tau_{k}^{\delta(m)} \wedge T}-\left\langle W_{i}^{\delta(n)}-W_{i}^{\delta(m)}\right\rangle_{\sigma_{k}^{\delta(m)} \wedge T}\right]\right] \\
& +E^{z}\left[\sum_{k=1}^{\infty}\left[\left\langle W_{i}^{\delta(n)}-W_{i}^{\delta(m)}\right\rangle_{\sigma_{k}^{\delta(m)} \wedge T}-\left\langle W_{i}^{\delta(n)}-W_{i}^{\delta(m)}\right\rangle_{\tau_{k-1}^{\delta(m)} \wedge T}\right]\right]
\end{aligned}
$$

We analyze each of the terms on the right hand side above separately. Regarding the first term one can see easily that for each $k \geq 1$ and $n>m \geq 1$ there exists an $\ell \geq 1$ such that $\left[\sigma_{k}^{\delta(m)}, \tau_{k}^{\delta(m)}\right] \subseteq\left[\sigma_{\ell}^{\delta(n)}, \tau_{\ell}^{\delta(n)}\right]$. It then follows that for $k \geq 1$ and $n>m$,

$$
W_{i}^{\delta(m)}\left(T \wedge \tau_{k}^{\delta(m)}\right)-W_{i}^{\delta(m)}\left(T \wedge \sigma_{k}^{\delta(m)}\right)=W_{i}^{\delta(n)}\left(T \wedge \tau_{k}^{\delta(m)}\right)-W_{i}^{\delta(n)}\left(T \wedge \sigma_{k}^{\delta(m)}\right),
$$




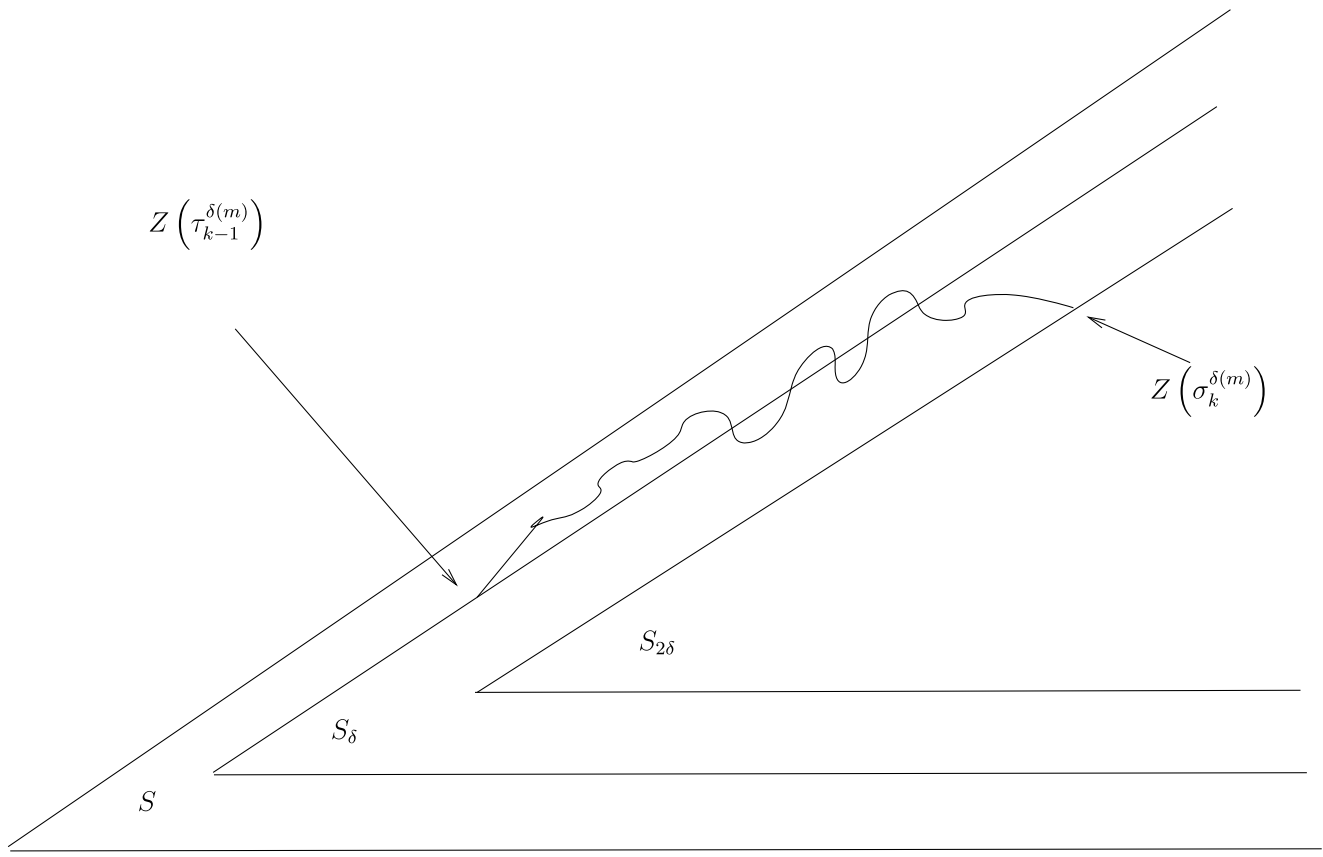

Fig. 3. If $t \in\left(\tau_{k-1}^{\delta(m)}, \sigma_{k}^{\delta(m)}\right)$ then $Z(t) \in S \backslash S_{2 \delta(m)}$.

hence by Proposition IV.1.13 in [25] the first term is zero. Regarding the second term note that by (16) for every $\delta>0$, $0 \leq s<t$, and $i=1,2$ we have $\left\langle W_{i}^{\delta}\right\rangle_{t}-\left\langle W_{i}^{\delta}\right\rangle_{s} \leq t-s$, and on the interval $\left[\tau_{k-1}^{\delta(m)}, \sigma_{k}^{\delta(m)}\right]$ the process $W^{\delta(m)}$ is flat. Then it follows that for $i=1,2$, we have

$$
\begin{aligned}
\sum_{k=1}^{\infty}\left[\left\langle W_{i}^{\delta(n)}-W_{i}^{\delta(m)}\right\rangle_{\sigma_{k}^{\delta(m)} \wedge T}-\left\langle W_{i}^{\delta(n)}-W_{i}^{\delta(m)}\right\rangle_{\tau_{k-1}^{\delta(m)} \wedge T}\right] & =\sum_{k=1}^{\infty}\left[\left\langle W_{i}^{\delta(n)}\right\rangle_{\sigma_{k}^{\delta(m)} \wedge T}-\left\langle W_{i}^{\delta(n)}\right\rangle_{\tau_{k-1}^{\delta(m)} \wedge T}\right] \\
& \leq \sum_{k=1}^{\infty}\left[\left(\sigma_{k}^{\delta(m)} \wedge T\right)-\left(\tau_{k-1}^{\delta(m)} \wedge T\right)\right] .
\end{aligned}
$$

Now note that if $t \in\left(\tau_{k-1}^{\delta(m)}, \sigma_{k}^{\delta(m)}\right)$ for some $k \geq 1$ then $Z(t) \in S \backslash S_{2 \delta(m)}$ (see Figure 3), thus

$$
\sum_{k=1}^{\infty}\left[\left(\sigma_{k}^{\delta(m)} \wedge T\right)-\left(\tau_{k-1}^{\delta(m)} \wedge T\right)\right]=\int_{0}^{T} \sum_{k=1}^{\infty} 1\left\{t \in\left(\tau_{k-1}^{\delta(m)}, \sigma_{k}^{\delta(m)}\right)\right\} d t \leq \int_{0}^{T} 1\left\{Z(t) \in S \backslash S_{2 \delta(m)}\right\} d t,
$$

and (17) follows.

Proposition 4.7. There exists a process $W$ on $\left(C_{S}, \mathcal{F}, \mathcal{F}_{t}\right)$ such that for every sequence $\{\delta(n), n \geq 1\} \in \Gamma$ and every $z \in S$, the process $W$ is a standard 2-dimensional Brownian motion under $P^{z}$ started at zero, and

$$
\lim _{n \rightarrow \infty} W_{i}^{\delta(n)}=W_{i}, \quad i=1,2,
$$

in the norm topology given in (15).

Proof. For every $z \in S$ a standard argument now shows the existence of a $P^{z}$-Brownian motion $W^{z}$ such that (20) holds with $W_{i}$ replaced by $W_{i}^{z}$. The existence of a single $W$ not depending on $z$ follows from Cinlar, Jacod, Protter, and Sharpe [9], Lemma 3.29. 
Proof of Part 1 of Theorem 4.2. We define

$$
X(t, \omega)=W(t, \omega)+\omega(0), \quad t \geq 0,
$$

and note that by Proposition 4.7 for every $z \in S$ the 2-dimensional process $X$ is indeed a standard Brownian motion on $\left(C_{S}, \mathcal{F}, \mathcal{F}_{t}, P^{z}\right)$ started at $z$, exactly as required.

\subsection{Proof of Part 2 of Theorem 4.2}

Let the set of continuous functions $\omega:[0, \infty) \mapsto \mathbb{R}^{2}$ be denoted by $C_{\mathbb{R}^{2}}$, which is an extension of $C_{S}$. On this space, for each $t \geq s \geq 0$, let $\tilde{\mathcal{M}}_{t}=\sigma\{\omega(s): 0 \leq s \leq t\}$, the sigma-field of subsets of $C_{\mathbb{R}^{2}}$ generated by the coordinate maps $\omega \mapsto \omega(s)$ for $0 \leq s \leq t$. Similarly, define $\overline{\tilde{\mathcal{M}}}=\sigma\{\omega(s): s \in[0, \infty)\}$. As explained in Williams [30], these $\sigma$-fields represent the natural extensions of $\mathcal{M}_{t}$ and $\mathcal{M}$ from $C_{S}$ to $C_{\mathbb{R}^{2}}$. Let $\tilde{X}:[0, \infty) \times C_{\mathbb{R}^{2}} \mapsto \mathbb{R}^{2}$ be the coordinatemapping process

$$
\tilde{X}(t, \omega)=\omega(t), \quad t \geq 0, w \in C_{\mathbb{R}^{2}} .
$$

For each $z \in S$, let $Q^{z}$ be a probability measure on $\tilde{\mathcal{M}}$ such that $\tilde{X}$ is a Brownian motion starting at $z$ under $Q^{z}$. Recall that $v_{1}$ and $v_{2}$ are the directions of reflection, and let $R$ be the $2 \times 2$ matrix whose column vectors are $v_{1}$ and $v_{2}$. The set $\mathcal{A}$ defined below will play a central role in this subsection.

Definition 4.8. Let $\mathcal{A} \subset C_{S} \times C_{\mathbb{R}^{2}}$ be the set that consists of all pairs $(z, y) \in C_{S} \times C_{\mathbb{R}^{2}}$ satisfying the following two properties:

1. There exists $u \in C_{\mathbb{R}^{2}}$ such that both components of $u$ are non-decreasing, and $\mathrm{y}(\mathrm{t})=\mathrm{Ru}(\mathrm{t})$ for all $t \geq 0$.

2. For $j=1,2$, the $j$ th component $u_{j}$ of $u$ increases only at times $t \geq 0$ when $z(t) \in \partial S^{j}$, i.e., $\int_{0}^{\infty} 1\{z(v) \in S \backslash$ $\left.\partial S^{j}\right\} d u_{j}(v)=0$.

One can show that the set $\mathcal{A}$ is measurable, that is, $\mathcal{A} \in \mathcal{M} \times \tilde{\mathcal{M}}$, but for brevity we omit the proof.

The next proposition is a restatement of the results on page 163 and of Theorem 1 of Williams [30].

Proposition 4.9. There exists a unique pair $(\tilde{Z}, \tilde{Y})$ of continuous, adapted processes on $\left(C_{\mathbb{R}^{2}}, \tilde{\mathcal{M}}, \tilde{\mathcal{M}} t\right)$ (both $\tilde{Z}$ and $\tilde{Y}$ are two-dimensional) such that

$$
\begin{aligned}
& \tilde{Y}(0)=0, \quad \tilde{Z}(t) \in S \quad \text { for each } t \geq 0, \\
& \tilde{Y}\left(t \vee \tilde{\tau}_{0}\right)=\tilde{Y}\left(\tilde{\tau}_{0}\right), \quad t \geq 0, \\
& \tilde{Z}(t)= \begin{cases}\tilde{X}(t)+\tilde{Y}(t) & \text { if } t \leq \tilde{\tau}_{0}, \\
0 & \text { if } t \geq \tilde{\tau}_{0},\end{cases}
\end{aligned}
$$

where $\tilde{\tau}_{0}=\inf \{t \geq 0: \tilde{Z}(t)=0\}$,

$$
(\tilde{Z}, \tilde{Y}) \in \mathcal{A} \text {. }
$$

Furthermore, for every $z \in S, Z\left(\cdot \wedge \tau_{0}\right)$ has the same law under $P^{z}$ as $\tilde{Z}\left(\cdot \wedge \tilde{\tau}_{0}\right)=\tilde{Z}(\cdot)$ has under $Q^{z}$, i.e., for any $B \in \mathcal{M}$

$$
P^{z}\left(Z\left(\cdot \wedge \tau_{0}\right) \in B\right)=Q^{z}\left(\tilde{Z}\left(\cdot \wedge \tilde{\tau}_{0}\right) \in B\right)
$$

Recall now the definitions of the stopping times $\tau_{k}^{\delta}, \sigma_{k}^{\delta}$ from the beginning of Section 4 . Since $Z$ is the coordinatemapping process, these definitions could be cast in the form $\sigma_{k}^{\delta}(w)=\inf \left\{t \geq \tau_{k-1}^{\delta}: w(t) \in S_{2 \delta}\right\}$ and $\tau_{k}^{\delta}(w)=\inf \{t \geq$ $\left.\sigma_{k}^{\delta}: w(t) \in \partial S_{\delta}\right\}$ for $w \in C_{S}$. We can also write $\tau_{0}=\inf \{t>0: w(t)=0\}$ for $w \in C_{S}$. In principle, some of these 
stopping times may be infinity for some $w \in S$, but by the continuity of $\omega$ we have $\lim _{k \rightarrow \infty} \sigma_{k}^{\delta}(\omega)=\lim _{k \rightarrow \infty} \tau_{k}^{\delta}(\omega)=$ $\infty$ for all $\omega \in C_{S}$. For $\delta>0$, we define the measurable mapping $I_{\delta}: C_{S} \mapsto C_{\mathbb{R}^{2}}$ as

$$
I_{\delta}(\omega)(\cdot)=\sum_{k=1}^{\infty}\left[\omega\left(\cdot \wedge \tau_{k}^{\delta}(\omega)\right)-\omega\left(\cdot \wedge \sigma_{k}^{\delta}(\omega)\right)\right] .
$$

By the continuity of $\omega$, for any $t \geq 0$, in the infinite sum defining $I_{\delta}(\omega)(t)$ all terms except finitely many are zero. We also note that

$$
I_{\delta}(\omega)(\cdot)=W^{\delta}(\cdot, \omega)
$$

Now let $\underline{0}$ be the zero function in $C_{\mathbb{R}^{2}}$.

Definition 4.10. For any given sequence $\{\gamma(n), n \geq 1\} \in \Gamma$, we define the mapping $F_{\gamma}: C_{S} \mapsto C_{\mathbb{R}^{2}}$ in the following way: if there exists a function $\tilde{\omega} \in C_{\mathbb{R}^{2}}$ such that for each $m \in \mathbb{N}_{+}$,

$$
\sup _{t \leq m}\left\|I_{\gamma(n)}(\omega)(t)-\tilde{\omega}(t)\right\|^{2} \rightarrow 0 \quad \text { as } n \rightarrow \infty,
$$

then $F_{\gamma}(\omega)=\tilde{\omega}$. If such $\tilde{\omega}$ does not exist, then let $F_{\gamma}(\omega)$ be $\underline{0}$.

It follows from part $\mathrm{R}$ on page 111 in [4], that $F_{\gamma}$ is $\mathcal{M} / \tilde{\mathcal{M}}$ measurable.

A few rather obvious properties of $I_{\delta}(\cdot)$ and $F_{\gamma}(\cdot)$ will be listed in the Appendix, see Lemmas A.1, A.2, A.3, and A.4.

Recall the process $W$ from Proposition 4.7 which also appears implicitly in (3) via (21). The following lemma is instrumental for the developments in this section. It specifies the exact form of the dependence of $W(\cdot, \omega)$ on $\omega$.

Lemma 4.11. For every $z \in S$ and $\{\delta(n), n \geq 1\} \in \Gamma$ there exists a sequence $\left\{\gamma^{z}(n), n \geq 1\right\} \subset\{\delta(n), n \geq 1\}$ such that

$$
P^{z}\left(F_{\gamma^{z}}(\omega)=W(\cdot, \omega)\right)=1 .
$$

In addition, the following three statements hold:

(a) If for some $z \in S$ and $\{\gamma(n), n \geq 1\} \in \Gamma$ we have $P^{z}\left(F_{\gamma}(\omega)=\underline{0}\right)=0$ then

$$
P^{z}\left(F_{\gamma}(\omega)=W(\cdot, \omega)\right)=1 ;
$$

(b) For every stopping time $\tau$ on $\left(C_{s}, \mathcal{F}, \mathcal{F}_{t}\right)$ and $z \in S$,

$$
E^{z}\left[P^{Z(\tau)}\left(F_{\gamma^{z}}(\omega)=W(\cdot, \omega)\right)\right]=1 ;
$$

(c) For every $z \in S$,

$$
P^{0}\left(F_{\gamma^{z}}(\omega)=W(\cdot, \omega)\right)=1 .
$$

Proof. Let $\{\delta(n), n \geq 1\} \in \Gamma$ be arbitrary. By the concavity of the function $x \mapsto \sqrt{x} \wedge 1$, (25), Doob's Maximal Inequality, and (20)

$$
\begin{aligned}
& \lim _{n \rightarrow \infty} \sum_{m=1}^{\infty} \frac{1}{2^{m}} E^{z}\left[\sup _{t \leq m}\left\{\left\|I_{\delta(n)}(\omega)(t)-W(t, \omega)\right\| \wedge 1\right\}\right] \\
& \quad \leq \lim _{n \rightarrow \infty} \sum_{m=1}^{\infty} \frac{1}{2^{m}}\left\{\left(E^{z}\left[\sup _{t \leq m}\left\{\left\|I_{\delta(n)}(\omega)(t)-W(t, \omega)\right\|^{2}\right\}\right]\right)^{1 / 2} \wedge 1\right\}
\end{aligned}
$$




$$
\begin{aligned}
& \leq \lim _{n \rightarrow \infty} \sum_{m=1}^{\infty} \frac{1}{2^{m}}\left\{\left(E^{z}\left[\sup _{t \leq m}\left\{\left\|W^{\delta(n)}(t, \omega)-W(t, \omega)\right\|^{2}\right\}\right]\right)^{1 / 2} \wedge 1\right\} \\
& \leq \lim _{n \rightarrow \infty} \sum_{m=1}^{\infty} \frac{1}{2^{m}}\left\{\left(4 \sum_{i=1,2} E^{z}\left[\left(W_{i}^{\delta(n)}(m)-W_{i}(m)\right)^{2}\right]\right)^{1 / 2} \wedge 1\right\} \\
& =0 .
\end{aligned}
$$

Then there exists a subsequence $\left\{\gamma^{z}(n), n \geq 1\right\} \subset\{\delta(n), n \geq 1\}$ such that

$$
\lim _{n \rightarrow \infty} \sum_{m=1}^{\infty} \frac{1}{2^{m}} \sup _{t \leq m}\left\{\left\|I_{\gamma^{z}(n)}(\omega)(t)-W(t, \omega)\right\| \wedge 1\right\}=0,
$$

$P^{z}$-a.s., which implies (26). Next we are going to show the statement in (a). From our assumption that $P^{z}\left(F_{\gamma}(\omega)=\right.$ $\underline{0})=0$, it follows that $\lim _{n \rightarrow \infty} I_{\gamma(n)}(\omega)=F_{\gamma}(\omega)$ in the topology of uniform convergence on compacts, $P^{z}$-a.s. On the other hand, similarly to (30) one can show that

$$
\lim _{n \rightarrow \infty} \sum_{m=1}^{\infty} \frac{1}{2^{m}} E^{z}\left[\sup _{t \leq m}\left\{\left\|I_{\gamma(n)}(\omega)(t)-W(t, \omega)\right\| \wedge 1\right\}\right]=0,
$$

which implies (27). Next we are going to show part (b). By (70) and (26) we have $P^{z}$-a.s.

$$
F_{\gamma^{z}}(\omega(\tau+\cdot))=F_{\gamma^{z}}(\omega)(\tau+\cdot)-F_{\gamma^{z}}(\omega)(\tau)=W(\tau+\cdot)-W(\tau) \neq \underline{0},
$$

thus by the strong Markov property

$$
1=P^{z}\left(F_{\gamma^{z}}(\omega(\tau+\cdot)) \neq \underline{0}\right)=P^{z}\left(F_{\gamma^{z}}(Z(\tau+\cdot)) \neq \underline{0}\right)=E^{z}\left[P^{Z(\tau)}\left(F_{\gamma^{z}}(Z(\cdot)) \neq \underline{0}\right)\right] .
$$

Therefore there exists a set $S^{\tau} \in \mathcal{B}_{S}$ such that $P^{z}\left(Z(\tau) \in S^{\tau}\right)=1$ and

$$
P^{z^{\prime}}\left(F_{\gamma^{z}}(Z(\cdot)) \neq \underline{0}\right)=1 \quad \text { for all } z^{\prime} \in S^{\tau}
$$

( $S^{\tau}$ is a Borel set by Varadhan and Williams [28], Corollary 3.3). Then by part (a) we have $P^{z^{\prime}}\left(F_{\gamma^{z}}(\omega)=\right.$ $W(\cdot, \omega))=1$ for all $z^{\prime} \in S^{\tau}$, which implies (28). Finally we show part (c). By part (a) all we need to show is that $P^{0}\left(F_{\gamma^{z}}(\omega)=\underline{0}\right)=0$. By the strong Markov property the left-hand side is equal to $P^{z}\left(F_{\gamma^{z}}\left(Z\left(\tau_{0}+\cdot\right)\right)=\underline{0}\right)$, which is indeed 0 by (31).

The following proposition studies the behavior of the pair $(Z, Y)$ on the interval $\left[0, \tau_{0}\right]$. A similar result may be derived using the combination of Theorem 3 in [20], Theorem 2.9 in [23] and Theorem 1 in [30]. However, the Brownian motion in [20] is defined differently than it is defined in the present paper, and so we present a proof below utilizing the functional relationship (26) between $Z(\omega)=\omega$ and $W(\omega)$. This functional relationship will be essential in studying the behavior of $(Z, Y)$ over excursion intervals of $Z$ away from the origin. Also, we note that for the proof of (26) (and for the other statements in Lemma 4.11) it is important that our definition of $X$ differs from that in [20].

For every $z \in S$, we fix a sequence $\left\{\gamma^{z}(n), n \geq 1\right\}$ such that (26) holds. Recall the class $\mathcal{A}$ from Definition 4.8.

Proposition 4.12. For each $z \in S$, we have $P^{z}$-a.s. that $\left(Z\left(\cdot \wedge \tau_{0}\right), Y\left(\cdot \wedge \tau_{0}\right)\right) \in \mathcal{A}$.

Proof. (26) implies that for any subsequence $\{\alpha(n), n \geq 1\} \subset\left\{\gamma^{z}(n), n \geq 1\right\}$ we have $F_{\alpha}(\omega)=W(\cdot, \omega), P^{z}$-a.s., and by $(67)$ and by $Z(\cdot, \omega)=\omega$ we have

$$
F_{\alpha}\left(Z\left(\cdot \wedge \tau_{0}\right)\right)=W\left(\cdot \wedge \tau_{0}\right)=X\left(\cdot \wedge \tau_{0}\right)-z,
$$


$P^{z}$-a.s. Then it is sufficient to show that there exists a sequence $\{\alpha(n), n \geq 1\} \subset\left\{\gamma^{z}(n), n \geq 1\right\}$ such that

$$
F_{\alpha}(\tilde{Z}(\cdot))=\tilde{X}\left(\cdot \wedge \tilde{\tau}_{0}\right)-z, \quad Q^{z} \text {-a.s. }
$$

Indeed, suppose that (32) holds, then

$$
\begin{aligned}
(\tilde{Z}(\cdot), \tilde{Y}(\cdot)) & =\left(\tilde{Z}(\cdot), \tilde{Z}(\cdot)-\tilde{X}\left(\cdot \wedge \tilde{\tau}_{0}\right)\right)=\left(\tilde{Z}(\cdot), \tilde{Z}(\cdot)-F_{\alpha}(\tilde{Z}(\cdot))-z\right) \\
& \stackrel{\mathrm{d}}{=}\left(Z\left(\cdot \wedge \tau_{0}\right), Z\left(\cdot \wedge \tau_{0}\right)-F_{\alpha}\left(Z\left(\cdot \wedge \tau_{0}\right)\right)-z\right)=\left(Z\left(\cdot \wedge \tau_{0}\right), Y\left(\cdot \wedge \tau_{0}\right)\right),
\end{aligned}
$$

where the sign $\stackrel{\mathrm{d}}{=}$ means that the law of the expression on the left-hand side under $Q^{z}$ agrees with the law of the expression on the right-hand side under $P^{z}$. Then the statement of the present proposition follows from Proposition 4.9. For the rest of this proof we shall prove (32) which amounts to showing that for some $\{\alpha(n), n \geq 1\} \subset\left\{\gamma^{z}(n), n \geq 1\right\}$ and every $m \in \mathbb{N}_{+}$we have

$$
\sup _{t \leq m}\left\|I_{\alpha(n)}(\tilde{Z}(\cdot))(t)-\left(\tilde{X}\left(t \wedge \tilde{\tau}_{0}\right)-z\right)\right\|^{2} \rightarrow 0
$$

as $n \rightarrow \infty, Q^{z}$-a.s.

Analog to $\tau_{0}^{\delta}, \tau_{1}^{\delta}, \sigma_{1}^{\delta}, \tau_{2}^{\delta}, \sigma_{2}^{\delta}, \ldots$ for $\delta>0$ we define the stopping times $\tilde{\tau}_{0}^{\delta}=0, \tilde{\sigma}_{k}^{\delta}=\inf \left\{t \geq \tilde{\tau}_{k-1}^{\delta}: \tilde{Z}(t) \in S_{2 \delta}\right\}$ and $\tilde{\tau}_{k}^{\delta}=\inf \left\{t \geq \tilde{\sigma}_{k}^{\delta}: \tilde{Z}(t) \in \partial S_{\delta}\right\}, k \geq 1$. In the rest of the proof we shall drop the superscript $z$ from $\gamma^{z}(n)$ whenever it leads to double superscript, and write $\tilde{\sigma}^{\gamma(n)}$ and $\tilde{\tau}^{\gamma(n)}$ instead.

By the fact that $\tilde{Z}$ is flat on $\left[\tilde{\tau}_{0}, \infty\right)$, we have

$$
I_{\gamma^{z}(n)}(\tilde{Z}(\cdot))(t \wedge m)=\sum_{k=1}^{\infty}\left[\tilde{Z}\left(t \wedge \tilde{\tau}_{0} \wedge \tilde{\tau}_{k}^{\gamma(n)} \wedge m\right)-\tilde{Z}\left(t \wedge \tilde{\tau}_{0} \wedge \tilde{\sigma}_{k}^{\gamma(n)} \wedge m\right)\right] .
$$

Moreover, since $\tilde{Y}$ is flat on $\left[\tilde{\sigma}_{k}^{\delta} \wedge m, \tilde{\tau}_{k}^{\delta} \wedge m\right]$, we can cast this in the form

$$
\begin{aligned}
I_{\gamma^{z}(n)}(\tilde{Z}(\cdot))(t \wedge m) & =\sum_{k=1}^{\infty}\left[\tilde{X}\left(t \wedge \tilde{\tau}_{0} \wedge \tilde{\tau}_{k}^{\gamma(n)} \wedge m\right)-\tilde{X}\left(t \wedge \tilde{\tau}_{0} \wedge \tilde{\sigma}_{k}^{\gamma(n)} \wedge m\right)\right] \\
& =\int_{0}^{t} \sum_{k=1}^{\infty} 1\left\{u \in\left[\tilde{\sigma}_{k}^{\gamma(n)} \wedge \tilde{\tau}_{0} \wedge m, \tilde{\tau}_{k}^{\gamma(n)} \wedge \tilde{\tau}_{0} \wedge m\right]\right\} d \tilde{X}(u) .
\end{aligned}
$$

Hence by the the Dominated Convergence Theorem, the fact that $Z\left(\cdot \wedge \tau_{0}\right)$ has the same law as $\tilde{Z}\left(\cdot \wedge \tilde{\tau}_{0}\right)$, and by Williams [29], Lemma 4.2, it follows that

$$
\begin{aligned}
& \lim _{n \rightarrow \infty} E_{Q}^{z}\left[\left\|I_{\gamma(n)}(\tilde{Z}(\cdot))\left(\tilde{\tau}_{0} \wedge m\right)-\left(\tilde{X}\left(\tilde{\tau}_{0} \wedge m\right)-z\right)\right\|^{2}\right] \\
& =2 \lim _{n \rightarrow \infty} E_{Q}^{z}\left[\sum_{k=0}^{\infty} \int_{0}^{\tilde{\tau}_{0} \wedge m} 1\left\{u \in\left[\tilde{\tau}_{k}^{\gamma(n)}, \tilde{\sigma}_{k+1}^{\gamma(n)}\right]\right\} d u\right] \\
& \quad \leq 2 \lim _{n \rightarrow \infty} E_{Q}^{z}\left[\int_{0}^{\tilde{\tau}_{0} \wedge m} 1\left\{\tilde{Z}(u) \in S \backslash S_{2 \gamma^{z}(n)}\right\} d u\right] \\
& =2 E_{Q}^{z}\left[\int_{0}^{\tilde{\tau}_{0} \wedge m} 1\{\tilde{Z}(u) \in \partial S\} d u\right] \\
& =2 E^{z}\left[\int_{0}^{\tau_{0} \wedge m} 1\{Z(u) \in \partial S\} d u\right] \\
& =0 .
\end{aligned}
$$


Then Doob's inequality applied to the square-integrable martingale $I_{\gamma(n)}(\tilde{Z} \wedge m)(\cdot)-\left(\tilde{X}\left(\cdot \wedge \tilde{\tau}_{0} \wedge m\right)-z\right)$ gives

$$
\begin{aligned}
& E_{Q}^{z} {\left[\sup _{t \leq m}\left\{\left\|I_{\gamma(n)}(\tilde{Z}(\cdot))\left(t \wedge \tilde{\tau}_{0}\right)-\left(\tilde{X}\left(t \wedge \tilde{\tau}_{0}\right)-z\right)\right\|^{2}\right\}\right] } \\
& \quad \leq 4 E_{Q}^{z}\left[\left\|I_{\gamma(n)}(\tilde{Z}(\cdot))\left(\tilde{\tau}_{0} \wedge m\right)-\left(\tilde{X}\left(\tilde{\tau}_{0} \wedge m\right)-z\right)\right\|^{2}\right] \rightarrow 0, \quad \text { as } n \rightarrow \infty,
\end{aligned}
$$

and so the existence of a subsequence $\{\alpha(n), n \geq 1\} \subset\left\{\gamma^{z}(n), n \geq 1\right\}$ follows such that (33) holds.

Proposition 4.13. For each $z \in S$, and each excursion interval $\left[G_{i}, D_{i}\right]$ of $Z$ away from the origin, we have $P^{z}$-a.s. that

$$
\left(Z\left(\left(G_{i}+\cdot\right) \wedge D_{i}\right), Y\left(\left(G_{i}+\cdot\right) \wedge D_{i}\right)\right) \in \mathcal{A} .
$$

Proof. Let $\left\{\left(U_{k}^{n}, T_{k}^{n}\right), k \geq 1\right\}$ be the sequence of excursion intervals of $Z$ away from zero with length strictly larger than $1 / n$, i.e., $T_{k}^{n}-U_{k}^{n}>1 / n$ for every $n \in \mathbb{N}_{+}$, and let $V_{k}^{n}=U_{k}^{n}+1 / n$. Clearly it is sufficient to show that for all $k, n \in \mathbb{N}_{+}$and $z \in S$,

$$
P^{z}\left(\left(Z\left(\left(V_{k}^{n}+\cdot\right) \wedge T_{k}^{n}\right), Y\left(\left(V_{k}^{n}+\cdot\right) \wedge T_{k}^{n}\right)\right) \in \mathcal{A}\right)=1
$$

which is the same as

$$
P^{z}\left(\left(Z\left(\left(V_{k}^{n}+\cdot\right) \wedge T_{k}^{n}\right), Z\left(\left(V_{k}^{n}+\cdot\right) \wedge T_{k}^{n}\right)-X\left(\left(V_{k}^{n}+\cdot\right) \wedge T_{k}^{n}\right)\right) \in \mathcal{A}\right)=1 .
$$

By (26) and (72) in the Appendix we have $P^{z}$-a.s. that

$$
X\left(\left(V_{k}^{n}+t\right) \wedge T_{k}^{n}\right)=F_{\gamma^{z}}(Z(\cdot))\left(\left(V_{k}^{n}+t\right) \wedge T_{k}^{n}\right)+z=F_{\gamma^{z}}\left(Z\left(\left(V_{k}^{n}+\cdot\right) \wedge T_{k}^{n}\right)\right)(t)+F_{\gamma^{z}}(Z(\cdot))\left(V_{k}^{n}\right)+z,
$$

hence we can replace the $X\left(\left(V_{k}^{n}+\cdot\right) \wedge T_{k}^{n}\right)$ term in (34) by $F_{\gamma^{z}}\left(Z\left(\left(V_{k}^{n}+\cdot\right) \wedge T_{k}^{n}\right)\right)$, since the terms $F_{\gamma^{z}}(Z(\cdot))\left(V_{k}^{n}\right)+z$ have no impact on the fact whether the pair $\left(Z\left(\left(V_{k}^{n}+\cdot\right) \wedge T_{k}^{n}\right), Z\left(\left(V_{k}^{n}+\cdot\right) \wedge T_{k}^{n}\right)-X\left(\left(V_{k}^{n}+\cdot\right) \wedge T_{k}^{n}\right)\right)$ is included in $\mathcal{A}$ or not. Thus the left-hand side of (34) is equal to

$$
P^{z}\left(\left(Z\left(\left(V_{k}^{n}+\cdot\right) \wedge T_{k}^{n}\right), Z\left(\left(V_{k}^{n}+\cdot\right) \wedge T_{k}^{n}\right)-F_{\gamma^{z}}\left(Z\left(\left(V_{k}^{n}+\cdot\right) \wedge T_{k}^{n}\right)\right) \in \mathcal{A}\right)\right),
$$

and by the strong Markov property and (67) this is equal to

$$
\begin{aligned}
& E^{z}\left[P^{Z\left(V_{k}^{n}\right)}\left(\left(Z\left(\cdot \wedge \tau_{0}\right), Z\left(\cdot \wedge \tau_{0}\right)-F_{\gamma^{z}}\left(Z\left(\cdot \wedge \tau_{0}\right)\right)\right) \in \mathcal{A}\right)\right] \\
& \quad=E^{z}\left[P^{Z\left(V_{k}^{n}\right)}\left(\left(Z\left(\cdot \wedge \tau_{0}\right), Z\left(\cdot \wedge \tau_{0}\right)-F_{\gamma^{z}}(Z(\cdot))\left(t \wedge \tau_{0}\right)\right) \in \mathcal{A}\right)\right] .
\end{aligned}
$$

When applying (67) above, we needed the condition $E^{z}\left[P^{Z\left(V_{k}^{n}\right)}\left(F_{\gamma^{z}}(Z(\cdot)) \neq \underline{0}\right)\right]$, but this follows from (28). Applying (28) and Proposition 4.12, we get that (35) is equal to

$$
E^{z}\left[P^{Z\left(V_{k}^{n}\right)}\left(\left(Z\left(\cdot \wedge \tau_{0}\right), Z\left(\cdot \wedge \tau_{0}\right)-X\left(\cdot \wedge \tau_{0}\right)\right) \in \mathcal{A}\right)\right]=1,
$$

as desired.

Proof of Part 2 of Theorem 4.2. Let $\left[G_{i}, D_{i}\right]$ be an arbitrary excursion interval of $Z(\cdot, \omega)$ away from zero. By Proposition 4.13 there exist two non-decreasing functions $U_{1}, U_{2}: \mathbb{R}_{+} \mapsto \mathbb{R}$, such that $Y\left(\left(G_{i}+t\right) \wedge D_{i}\right)=v_{1} U_{1}(t)+$ $v_{2} U_{2}(t)$ for $t \geq 0$, and

$$
\int_{0}^{\infty} 1\left\{Z\left(\left(G_{i}+p\right) \wedge D_{i}\right) \notin \partial S^{j}\right\} d U_{j}(p)=0
$$

for $j=1,2$. Then, by Lemma A.5 in the Appendix,

$$
V_{1}\left(Y\left(\left(G_{i}+\cdot\right) \wedge D_{i}\right),[0, t]\right)=\left\|v_{1}\right\|\left(U_{1}(t)-U_{1}(0)\right)+\left\|v_{2}\right\|\left(U_{2}(t)-U_{2}(0)\right), \quad t \geq 0 .
$$


The first three requirements of Definition 4.1 are obviously satisfied. Item 4 of Definition 4.1 follows from (36). Moreover, from (36) it follows that

$$
Y\left(\left(G_{i}+t\right) \wedge D_{i}\right)-Y\left(G_{i}\right)=\int_{0}^{t} \gamma(p) d\left(\left\|v_{1}\right\| U_{1}(p)+\left\|v_{2}\right\| U_{2}(p)\right)
$$

where

$$
\gamma(p)=\frac{v_{1}}{\left\|v_{1}\right\|} 1\left\{Z\left(\left(G_{i}+p\right) \wedge D_{i}\right) \in \partial S^{1}\right\}+\frac{v_{2}}{\left\|v_{2}\right\|} 1\left\{Z\left(\left(G_{i}+p\right) \wedge D_{i}\right) \in \partial S^{2}\right\},
$$

and so Item 5 of Definition 4.1 follows. Clearly the same proof applies if instead of $\left[G_{i}, D_{i}\right]$ we consider the interval $\left[0, \tau_{0}\right]$, whenever $\tau_{0}>0$; the only difference is that we have to use Proposition 4.12 instead of Proposition 4.13.

\section{Proof of Theorem 2.4}

Let the process $X$ be as in Theorem 4.2, so for each $z \in S$ the process $X$ is a Brownian motion started from $z$ on $\left(C_{S}, \mathcal{F}, \mathcal{F}_{t}, P^{z}\right)$, and let $Y$ be defined by $Z=X+Y$. In particular, $X$ is a local martingale on $\left(C_{S}, \mathcal{F}, \mathcal{F}_{t}, P^{z}\right)$. Hence, by Definition 2.3 of Section 2, in order to prove Theorem 2.4 it suffices to prove that for each $z \in S, Y$ is a continuous, zero-energy process (see Definition 2.2) on $\left(C_{S}, \mathcal{F}, \mathcal{F}_{t}, P^{z}\right)$ with $P^{z}(Y(0)=0)=1$. The fact that $Y$ is continuous with $P^{z}(Y(0)=0)=1$ is immediate since $Y=Z-X$, and so the proof of Theorem 2.4 is reduced to proving that $Y$ is of zero-energy on $\left(C_{S}, \mathcal{F}, \mathcal{F}_{t}, P^{z}\right)$.

Now let $E \subseteq \mathbb{R}_{+}$and $f: E \mapsto \mathbb{R}^{d}$. Then, for each $\gamma>0$ the function $f$ is said to be Hölder continuous with exponent $\gamma$ and Hölder constant $H(f) \in \mathbb{R}_{+}$, if

$$
\|f(t)-f(s)\| \leq H(f)|t-s|^{\gamma} \quad \text { for } s, t \in E .
$$

In addition, we say that $f$ is locally Hölder continuous on $\mathbb{R}_{+}$if it is Hölder continuous on compact $E \subset \mathbb{R}_{+}$. In order to prove that $Y$ is of zero-energy on $\left(C_{S}, \mathcal{F}, \mathcal{F}_{t}, P^{z}\right)$ for each $z \in S$, it suffices to show (see the proof of Theorem 2.4 below) that $P^{0}$-a.s. we have the decomposition $Y=g \circ \varphi$, where $\varphi: \mathbb{R}_{+} \mapsto \mathbb{R}_{+}$is a continuous, non-decreasing function and $g: \varphi\left(\mathbb{R}_{+}\right) \mapsto \mathbb{R}^{2}$ is a locally Hölder continuous function with Hölder exponent greater than $1 / 2$. The fact that such a decomposition exists is the content of the following proposition.

Proposition 5.1. Suppose that $\alpha<p<2$. Then, for $P^{0}$-a.e. $\omega \in C_{S}$, there exists a continuous, nondecreasing function $\varphi_{p}: \mathbb{R}_{+} \mapsto \mathbb{R}_{+}$and a map $g_{p}: \varphi_{p}\left(\mathbb{R}_{+}\right) \mapsto \mathbb{R}^{2}$, which is locally Hölder continuous with exponent $1 / p$, such that $Y(\cdot, \omega)=g_{p} \circ \varphi_{p}$ on $\mathbb{R}_{+}$.

In the following subsection, we provide the proof of Proposition 5.1. We close this subsection with the proof of Theorem 2.4.

Proof of Theorem 2.4. We first treat the case of $z=0$. By the decomposition $Z=X+Y$, where $X$ is a Brownian motion started from 0 on $\left(C_{S}, \mathcal{F}, \mathcal{F}_{t}, P^{0}\right)$, in order to show that $Z$ is a Dirichlet process on $\left(C_{S}, \mathcal{F}, \mathcal{F}_{t}, P^{0}\right)$, it suffices by Definition 2.3 to prove that $Y$ is a process of zero energy on $\left(C_{S}, \mathcal{F}, \mathcal{F}_{t}, P^{0}\right)$. Let $\alpha<p<2$ and recall by Proposition 5.1 that for $P^{0}$-a.e. $\omega \in C_{S}, Y(\cdot, \omega)=g_{p} \circ \varphi_{p}$ on $\mathbb{R}_{+}$, where $\varphi_{p}: \mathbb{R}_{+} \mapsto \mathbb{R}$ is a continuous, nondecreasing function and $g_{p}: \varphi_{p}(E) \mapsto \mathbb{R}^{2}$ is locally Hölder continuous on $\mathbb{R}_{+}$with exponent $1 / p$.

Now let $T \geq 0$ and let $H_{\varphi_{p}(T)}\left(g_{p}\right)$ denote the Hölder constant of $g_{p}$ on $\left[0, \varphi_{p}(T)\right]$. Then, for any partition $\pi$ of $[0, T]$,

$$
\begin{aligned}
\sum_{t_{i} \in \pi}\left\|Y\left(t_{i}\right)-Y\left(t_{i-1}\right)\right\|^{2} & =\sum_{t_{i} \in \pi}\left\|g_{p} \circ \varphi_{p}\left(t_{i}\right)-g_{p} \circ \varphi_{p}\left(t_{i-1}\right)\right\|^{2} \\
& \leq\left(H_{\varphi_{p}(T)}\left(g_{p}\right)\right)^{2} \sum_{t_{i} \in \pi}\left|\varphi_{p}\left(t_{i}\right)-\varphi_{p}\left(t_{i-1}\right)\right|^{2 / p} \\
& \leq\left(H_{\varphi_{p}(T)}\left(g_{p}\right)\right)^{2}\left(\varphi_{p}(T)-\varphi_{p}(0)\right) \max _{t_{i} \in \pi}\left|\varphi_{p}\left(t_{i}\right)-\varphi_{p}\left(t_{i-1}\right)\right|^{2 / p-1} .
\end{aligned}
$$


Next let $\left\{\pi^{n}, n \geq 1\right\}$ be a sequence of partitions of $[0, T]$ with $\left\|\pi^{n}\right\| \rightarrow 0$ as $n \rightarrow \infty$, and note that since $\alpha<p<2$, $2 / p-1>0$. Then, since $\varphi_{p}$ is $P^{0}$-a.s. continuous, it follows that $\max _{t_{i} \in \pi^{n}}\left|\varphi_{p}\left(t_{i}\right)-\varphi_{p}\left(t_{i-1}\right)\right|^{2 / p-1} \rightarrow 0$ as $n \rightarrow$ $\infty, P^{0}$-a.s., and so by the above

$$
\sum_{t_{i} \in \pi}\left\|Y\left(t_{i}\right)-Y\left(t_{i-1}\right)\right\|^{2} \rightarrow 0 \quad \text { as } n \rightarrow \infty, P^{0} \text {-a.s. }
$$

which implies that $Y$ is of zero-energy on $\left(C_{S}, \mathcal{F}, \mathcal{F}_{t}, P^{0}\right)$. Thus, $Z$ is a Dirichlet process on $\left(C_{S}, \mathcal{F}, \mathcal{F}_{t}, P^{0}\right)$.

Now let $z \in S$ arbitrary. Again by the decomposition $Z=X+Y$, where $X$ is a Brownian motion started from $z$ on $\left(C_{S}, \mathcal{F}, \mathcal{F}_{t}, P^{z}\right)$ it suffices by Definition 2.3 to prove that $Y$ is a process of zero energy on $\left(C_{S}, \mathcal{F}, \mathcal{F}_{t}, P^{z}\right)$. By the arguments of the previous portion of the proof, it then suffices to show that for $P^{z}$-a.e. $\omega \in C_{S}$, we have for each $\alpha<p<2$ the decomposition $Y(\cdot, \omega)=g_{p} \circ \varphi_{p}$ on $\mathbb{R}_{+}$, where $\varphi_{p}: \mathbb{R}_{+} \mapsto \mathbb{R}$ is a continuous, nondecreasing function and $g_{p}: \varphi_{p}(E) \mapsto \mathbb{R}^{2}$ is locally Hölder continuous on $\mathbb{R}_{+}$with exponent $1 / p$. However, by Theorem 3.1 of Chistyakov and Galkin [7] this will be implied if for each $\alpha<p<2$ we have that

$$
P^{z}\left(V_{p}(Y,[0, T])<\infty\right)=1, \quad T \geq 0 .
$$

Recall from (9) of Section 4 the definition of the stopping time $\tau_{0}=\inf \{t>0: Z(t)=0\}$. By Theorem 2.2 of Varadhan amd Williams [28], since $\alpha>0, P^{z}\left(\tau_{0}<\infty\right)=1$. By the monotonicity property of $V_{p}$ (see (P2) in Chistyakov and Galkin [7]),

$$
P^{z}\left(V_{p}(Y,[0, T])<\infty\right) \geq P^{z}\left(V_{p}\left(Y,\left[0, \tau_{0}+T\right]\right)<\infty\right) .
$$

By the semi-additivity property (see (P3) in Chistyakov and Galkin [7]) the right-hand side in the above equation is equal to

$$
P^{z}\left(V_{p}\left(Y,\left[0, \tau_{0}\right]\right)<\infty \quad \text { and } \quad V_{p}\left(Y,\left[\tau_{0}, \tau_{0}+T\right]\right)<\infty\right)
$$

Now using the strong Markov property [28], this expression can be written as

$$
\begin{aligned}
E^{z} & {\left[1\left\{V_{p}\left(Y,\left[0, \tau_{0}\right]\right)<\infty\right\} P^{z}\left(V_{p}\left(Y,\left[\tau_{0}, \tau_{0}+T\right]\right)<\infty \mid \mathcal{F}_{\tau_{0}}\right)\right] } \\
& =P^{z}\left(V_{p}\left(Y,\left[0, \tau_{0}\right]\right)<\infty\right) P^{0}\left(V_{p}(Y,[0, T])<\infty\right) .
\end{aligned}
$$

However, using Theorem 3.1 of Chistyakov and Galkin [7] it has already been shown earlier in the proof that the second factor in (38) is equal to 1 . By Part 2 of Theorem 4.2, $P^{z}\left(V_{1}\left(Y,\left[0, \tau_{0}\right]\right)<\infty\right)=1$, and this implies that the first term in (38) is also equal to 1 .

\subsection{Proof of Proposition 5.1}

In this section, we provide the proof of Proposition 5.1. For each $p>\alpha$ and $q>\alpha / 2$, define the function $\varphi_{p, q}: \mathbb{R}_{+} \mapsto$ $\mathbb{R}_{+}$by setting

$$
\varphi_{p, q}(t)= \begin{cases}V_{q}\left(L^{-1},[0, L(t)]\right), & \text { for } t \in L^{-1}\left(\mathbb{R}_{+}\right), \\ V_{q}\left(L^{-1},[0, L(t))\right), & \text { for } t \in \Lambda \backslash L^{-1}\left(\mathbb{R}_{+}\right), \\ \varphi_{p, q}\left(G_{i}\right)+\frac{\left(D_{i}-G_{i}\right)^{q}}{V_{p}\left(Y,\left[G_{i}, D_{i}\right]\right)} \cdot V_{p}\left(Y,\left[G_{i}, t\right]\right), & \text { for } t \in\left(G_{i}, D_{i}\right) \text { for } i \geq 1,\end{cases}
$$

where we recall from Section 3 the definition of $\Lambda$ as the zero set of $Z$, and of $L$ as the local time of $Z$ at the origin. Also recall from (8) of Section 3 the definition of the sequence $\left.\left\{\left(G_{i}, D_{i}\right), i \geq 1\right)\right\}$ of excursion intervals away from the origin of $Z$. Our proof of Proposition 5.1 will involve showing that for each $p>\alpha$ and $1>q>\alpha / 2$, we may $P^{0}$-a.s. write $Y(t)=Y \circ \varphi_{p, q}^{-1} \circ \varphi_{p, q}(t)$ for $t \in \mathbb{R}_{+}$. Moreover, it will be shown that $\varphi_{p, q}: \mathbb{R}_{+} \mapsto \mathbb{R}_{+}$is $P^{0}$-a.s. well-defined, non-decreasing and continuous with $\varphi_{p, q}\left(\mathbb{R}_{+}\right)=\mathbb{R}_{+}$, and that for each $p>\alpha$ there exists some $\alpha / 2<q<p / 2$ such that $Y \circ \varphi_{p, q}^{-1}$ is locally Hölder continuous on $\mathbb{R}_{+}$with Hölder exponent $1 / p$.

We begin with the following three preparatory lemmas. 
Lemma 5.2. For each $p>\alpha$ and $q>\alpha / 2$, the function $\varphi_{p, q}: \mathbb{R}_{+} \mapsto \mathbb{R}_{+}$is $P^{0}$-a.s. well-defined and strictly increasing on the set $\Lambda$. Moreover, if in addition to the above assumptions for $p$ and $q$ it is also true that $q<1$, then $P^{0}$-a.s.,

$$
\varphi_{p, q}(t)=\sum_{i: G_{i}<t}\left(D_{i}-G_{i}\right)^{q}, \quad t \in \Lambda .
$$

Proof. Let $p>\alpha$ and $q>\alpha / 2$. First note that by (8), it follows that $P^{0}$-a.s. the three cases provided in the definition of $\varphi_{p, q}$ are disjoint. Now recall from Theorem 2.6 of Williams [31] that $L^{-1}$ is a stable subordinator of index $\alpha / 2$ under $P^{0}$. Hence, by the results of Simon [27] (see immediately below display (9) of [27]), it follows that $P^{0}\left(V_{q}\left(L^{-1},[0,1]\right)<\infty\right)=1$. Using the scaling property [31] under $P^{0}$,

$$
L^{-1}(\lambda \cdot) \stackrel{\mathrm{d}}{=} \lambda^{2 / \alpha} L^{-1}(\cdot), \lambda>0,
$$

it then follows that $P^{0}\left(V_{q}\left(L^{-1},[0, T]\right)<\infty\right)=1$ for each $T \geq 0$. Moreover, by the monotonicity property of strong $q$-variation (see (P2) of Chistyakov and Galkin [7]), $V_{q}\left(L^{-1},[0, \cdot]\right)$ is $P^{0}$-a.s. a non-decreasing function. Hence, $P^{0}\left(V_{q}\left(L^{-1},[0, T]\right)<\infty, T \geq 0\right)=1$. It now follows from (39) that $\varphi_{p, q}$ is $P^{0}$-a.s. well-defined on the set $\Lambda$.

Next, we show (40). It follows from (11) of Section 3 and (9) of Simon [27] that for $\alpha / 2<q<1, P^{0}$-a.s.,

$$
V_{q}\left(L^{-1},[0, a]\right)=\sum_{i: L\left(G_{i}\right) \leq a}\left(D_{i}-G_{i}\right)^{q} \quad \text { and } \quad V_{q}\left(L^{-1},[0, a)\right)=\sum_{i: L\left(G_{i}\right)<a}\left(D_{i}-G_{i}\right)^{q}, \quad a \geq 0 .
$$

(40) now follows from (39) and (41), combined with the identity (12).

Finally, we show that $\varphi_{p, q}$ is $P^{0}$-a.s. strictly increasing on the set $\Lambda$. By Item 3 of Definition 2.1 , it follows that $P^{0}$-a.s. for every $t, t^{\prime} \in \Lambda$ such that $t<t^{\prime}$, there exists some $s \in\left(t, t^{\prime}\right)$ such that $s \notin \Lambda$. But then by (8), $G_{i} \in\left[t, t^{\prime}\right)$ for some $i \geq 1$, and so (40) implies $\varphi_{p, q}(t)<\varphi_{p, q}\left(t^{\prime}\right)$.

Lemma 5.3. For each $i \geq 1$ and $\varepsilon>0$,

$$
P^{0}\left(V_{1}\left(Y,\left[G_{i}, D_{i} \wedge\left(G_{i}+\varepsilon\right)\right]\right)>0\right)=1 .
$$

Proof. First, we need a slight generalization of Definition 2.9.10 and Theorem 2.9.12 in Karatzas and Shreve [21]. Let $f: \mathbb{R}_{+} \mapsto \mathbb{R}$ be given. A time $t \geq 0$ will be called a point of local maximum (minimum) from the right if there exists a $\delta>0$ such that $f(s) \leq f(t)(f(s) \geq f(t))$ for every $s \in(t, t+\delta)$. A time $t \geq 0$ will be called a point of strict local maximum (minimum) from the right if there exists a $\delta>0$ such that $f(s)<f(t)(f(s)>f(t))$ for every $s \in(t, t+\delta)$. Now let $w$ be a standard (1-dimensional) Brownian motion on some probability space. Then, following the proof of Theorem 2.9.12 in [21], for almost every sample path of $w$ all points of local maximum from the right are strict. In a similar manner, it is also true that for almost every path $w$ all points of local minimum from the right are strict.

Now we are ready to prove (42). We shall use the same notation as in Williams [31]. In particular, we adjoin a point $\partial$ to $C_{S}$, and define the excursion process $\left\{U_{t}, t \geq 0\right\}$ as the $\left(C_{S}, \mathcal{M}\right)$-valued point process given for $t \geq 0$ by

$$
U_{t}(s)= \begin{cases}Z\left(L^{-1}(t-)+s\right), & \text { if } 0 \leq s<L^{-1}(t)-L^{-1}(t-), \\ 0, & \text { if } s \geq L^{-1}(t)-L^{-1}(t-)>0, \\ \partial, & \text { if } L^{-1}(t)-L^{-1}(t-)=0 .\end{cases}
$$

Now let $N(A, U)$ be the number of times $t$ such that $\left(t, U_{t}\right) \in A$, where $A \in \mathcal{B} \times \mathcal{M}$ and $\mathcal{B}$ is the $\sigma$-field of Borel subsets of $(0, \infty)$. Then, from Theorem 3.3 of Williams [31] it follows that

$$
E^{0}\left[N\left((0, t) \times\left\{\omega: T_{\partial S}(\omega)>0\right\}, U\right)\right]=0,
$$

where $T_{\partial S}(\omega)=\inf \{t>0: \omega(t) \in \partial S\}$ for $\omega \in C_{S}$. Then, by the Monotone Convergence Theorem [22],

$$
E^{0}\left[N\left((0, \infty) \times\left\{\omega: T_{\partial S}(\omega)>0\right\}, U\right)\right]=0 .
$$


This implies that for $P^{0}$-a.e. sample path $T_{\partial S}^{i}=G_{i}$ for all $i \geq 1$, where

$$
T_{\partial S}^{i}=\inf \left\{t>G_{i}: Z(t) \in \partial S\right\} .
$$

Suppose now that (42) does not hold. That is, $Y$ is flat on $\left[G_{i},\left(G_{i}+\epsilon\right) \wedge D_{i}\right]$ for some $i \geq 1$. Since $Z\left(G_{i}\right)=0$,

$$
Z(t)=X(t)+Y\left(G_{i}\right)=X(t)-X\left(G_{i}\right), \quad t \in\left[G_{i},\left(G_{i}+\epsilon\right) \wedge D_{i}\right] .
$$

Now $T_{\partial S}^{i}=G_{i}$ implies the existence of a sequence $t_{n} \downarrow G_{i}$ such that $Z\left(t_{n}\right) \in \partial S^{1}$ for all $n \geq 1$, or $Z\left(t_{n}\right) \in \partial S^{2}$ for all $n \geq 1$. In the first case, $Z_{2}\left(t_{n}\right)=0$ for all $n \geq 1$. Thus, by (43), $G_{i}$ is a point of local minimum from the right for the Brownian motion $X_{2}$ (where $X_{2}$ is the second component of the two-dimensional Brownian motion $X$ ), but not strict, which is a contradiction. In the second case, we proceed similarly. $G_{i}$ is a point of local minimum from the right for the Brownian motion $\left\langle n_{2}, X\right\rangle$, but not strict, which is again a contradiction.

Lemma 5.4. For each $p>\alpha$ and $q>\alpha / 2$, the function $\varphi_{p, q}: \mathbb{R}_{+} \mapsto \mathbb{R}_{+}$is $P^{0}$-a.s. well-defined, non-decreasing and continuous on the set $\left(G_{i}, D_{i}\right)$ for $i \geq 1$.

Proof. Let $p>\alpha$ and $q>\alpha / 2$. By (8), it follows that $P^{0}$-a.s. the three cases provided in the definition of $\varphi_{p, q}$ are disjoint. We now show that $\varphi_{p, q}$ is $P^{0}$-a.s. well-defined on $\left(G_{i}, D_{i}\right)$ for each $i \geq 1$. By (39) and the fact that $V_{p}\left(Y,\left[G_{i}, \cdot\right]\right)$ is non-decreasing on $\left(G_{i}, D_{i}\right)$, it suffices to show that $P^{0}$-a.s., $0<V_{p}\left(Y,\left[G_{i}, D_{i}\right]\right)<+\infty$. The fact that $P^{0}$-a.s., $0<V_{p}\left(Y,\left[G_{i}, D_{i}\right]\right)$ follows by Lemma 5.3 above. The fact that $P^{0}$-a.s., $V_{p}\left(Y,\left[G_{i}, D_{i}\right]\right)<+\infty$ follows since by Part 2 of Theorem 4.2, $V_{1}\left(Y,\left[G_{i}, D_{i}\right]\right)<+\infty P^{0}$-a.s., and the fact that $V_{p}\left(Y,\left[G_{i}, D_{i}\right]\right) \leq\left(V_{1}\left(Y,\left[G_{i}, D_{i}\right]\right)\right)^{p}$ (see Remark 2.5 of Chistyakov and Galkin [7]) since by assumption $p>\alpha>1$. The fact that $\varphi_{p, q}: \mathbb{R}_{+} \mapsto \mathbb{R}_{+}$is $P^{0}$ a.s. continuous and non-decreasing on $\left(G_{i}, D_{i}\right)$ follows by (39) and the facts that $V_{p}\left(Y,\left[G_{i}, \cdot\right]\right)$ is non-decreasing on $\left(G_{i}, D_{i}\right)$ and, since $Y$ is $P^{0}$-a.s. continuous, $V_{p}\left(Y,\left[G_{i}, \cdot\right]\right)$ is $P^{0}$-a.s. continuous on $\left(G_{i}, D_{i}\right)$ as well.

Combining Lemmas 5.2 and 5.4 together, we may now prove the following.

Proposition 5.5. For each $p>\alpha$ and $1>q>\alpha / 2$, the function $\varphi_{p, q}: \mathbb{R}_{+} \mapsto \mathbb{R}_{+}$is $P^{0}$-a.s. well-defined, nondecreasing and continuous with $\varphi_{p, q}\left(\mathbb{R}_{+}\right)=\mathbb{R}_{+}$.

Proof. Let $p>\alpha$ and $1>q>\alpha / 2$. By (8), it follows that $P^{0}$-a.s. the three cases provided in the definition of $\varphi_{p, q}$ are disjoint and cover all of $\mathbb{R}_{+}$. The fact that $\varphi_{p, q}: \mathbb{R}_{+} \mapsto \mathbb{R}_{+}$is $P^{0}$-a.s. well-defined and non-decreasing now follows immediately by Lemmas 5.2 and 5.4 .

We next prove that $\varphi_{p, q}: \mathbb{R}_{+} \mapsto \mathbb{R}_{+}$is $P^{0}$-a.s. continuous. By Lemma 5.4, it suffices to show that $P^{0}$-a.s. $\varphi_{p, q}$ is continuous at each point $t \in \Lambda$. Let $\mathcal{H}_{1}$ be the set of all $\omega \in C_{S}$ such that the following four conditions hold,

1. Identity (8) holds,

2. Identity (40) holds,

3. $V_{p}\left(Y(\cdot, \omega),\left[G_{i}, \cdot\right]\right)$ is continuous on $\left(G_{i}, D_{i}\right)$ for each $i \geq 1$,

4. $\varphi_{p, q}(\cdot, \omega): \mathbb{R}_{+} \mapsto \mathbb{R}_{+}$is well-defined and non-decreasing.

By the discussion in Section 3, together with the definition of $\varphi_{p, q}$ and Lemmas 5.2 and 5.4, and the discussion of the preceding paragraph, it follows that $P^{0}\left(\mathcal{H}_{1}\right)=1$. It therefore suffices to show that $\varphi_{p, q}(\cdot, \omega): \mathbb{R}_{+} \mapsto \mathbb{R}_{+}$is continuous for each $\omega \in \mathcal{H}_{1}$. Hence, fix an arbitrary $\hat{\omega} \in \mathcal{H}_{1}$ for the remainder of this paragraph. Let $t \in \Lambda$ be arbitrary. We show that $\varphi_{p, q}$ is both left- and right-continuous at $t$. We start with left-continuity at $t$. Obviously then we can assume that $t>0$. If $t$ isolated from the left in $\Lambda$, then since identity (8) holds by condition $1, t=D_{i}$ for some $i \geq 1$, and so left-continuity follows from the fact that by condition $4, \varphi_{p, q}$ is well-defined, the continuity of $V_{p}\left(Y,\left[G_{i}, \cdot\right]\right)$ on $\left(G_{i}, D_{i}\right)$, which is guaranteed by condition 3, and the identity (40) guaranteed by condition 2 . Suppose now that $t$ is not isolated from the left in $\Lambda$, i.e. there exists a sequence $\left\{t_{n}, n \geq 1\right\} \subset \Lambda$ such that $t_{n} \uparrow t$ as $n \rightarrow \infty$. Then, since (40) holds by condition 2 , it follows that

$$
\lim _{n \rightarrow \infty}\left(\varphi_{p, q}(t)-\varphi_{p, q}\left(t_{n}\right)\right)=\lim _{n \rightarrow \infty} \sum_{i: t_{n} \leq G_{i}<t}\left(D_{i}-G_{i}\right)^{q}=0,
$$


and so left-continuity follows since by condition $4, \varphi_{p, q}$ is non-decreasing. The proof of right-continuity of $\varphi_{p, q}$ at $t$ is quite similar and so we omit the details.

We now complete the proof by showing that $P^{0}$-a.s., $\varphi_{p, q}\left(\mathbb{R}_{+}\right)=\mathbb{R}_{+}$. First note that using the fact from Section 3 that $L^{-1}$ is a subordinator under $P^{0}$, and the subadditivity property of strong $q$-variation (see (P3) in Section 2.2 of Chistyakov and Galkin [7]), it is straightforward to show that $P^{0}$-a.s., $V_{q}\left(L^{-1},[0, A]\right) \rightarrow+\infty$ as $A \rightarrow+\infty$. Hence, since by the discussion in Section 3, $P^{0}$-a.s., $L(t) \rightarrow+\infty$ as $t \rightarrow+\infty$, it follows by (39) and the fact that $\varphi_{p, q}: \mathbb{R}_{+} \mapsto \mathbb{R}_{+}$is $P^{0}$-a.s. non-decreasing, that $P^{0}$-a.s., $\varphi_{p, q}(t) \rightarrow+\infty$ as $t \rightarrow+\infty$. Moreover, it is clear from (39) that $P^{0}$-a.s., $\varphi_{p, q}(0)=0$. The desired result now follows since $\varphi_{p, q}$ is $P^{0}$-a.s. continuous on $\mathbb{R}_{+}$.

By Proposition 5.5 above, we now have that for each $p>\alpha$ and $1>q>\alpha / 2$ the function $\varphi_{p, q}: \mathbb{R}_{+} \mapsto \mathbb{R}_{+}$is $P^{0}$-a.s. well-defined, non-decreasing and continuous with $\varphi_{p, q}\left(\mathbb{R}_{+}\right)=\mathbb{R}_{+}$. Hence, we may $P^{0}$-a.s. define its rightcontinuous inverse function

$$
\varphi_{p, q}^{-1}(\tau)=\inf \left\{t \geq 0: \varphi_{p, q}(t)>\tau\right\}, \quad \tau \geq 0 .
$$

In preparation for the ultimate proof of Proposition 5.1 below, we first provide two lemmas regarding $\varphi_{p, q}^{-1}$.

Lemma 5.6. For each $p>\alpha$ and $1>q>\alpha / 2$, it follows that $P^{0}$-a.s., $\varphi_{p, q}^{-1} \circ \varphi_{p, q}(t)=t$ for $t \in \Lambda$. Moreover, $\varphi_{p, q}^{-1}$ is $P^{0}$-a.s. Hölder continuous on $\varphi_{p, q}(\Lambda)$ with Hölder exponent $1 / q$ and Hölder constant $H\left(\varphi_{p, q}^{-1}\right) \leq 1$.

Proof. Let $p>\alpha$ and $1>q>\alpha / 2$. Next, let $\mathcal{H}_{2}$ be the set of all $\omega \in C_{S}$ such that the following five conditions hold,

1. There are no isolated points in $\Lambda$ and the identities (8) and (12) hold,

2. $L^{-1}$ is strictly increasing and right-continuous,

3. $\varphi_{p, q}: \mathbb{R}_{+} \mapsto \mathbb{R}_{+}$is well-defined, non-decreasing and continuous with $\varphi_{p, q}\left(\mathbb{R}_{+}\right)=\mathbb{R}_{+}$,

4. $\varphi_{p, q}$ is strictly increasing on $\Lambda$,

5. $V_{1}\left(Y,\left[G_{i}, D_{i} \wedge\left(G_{i}+2^{-j}\right)\right]\right)>0$ for $i, j \geq 1$.

By the discussion in Section 3, Proposition 5.5, and Lemmas 5.2 and 5.3, it follows that $P^{0}\left(\mathcal{H}_{2}\right)=1$. It therefore suffices to show that the results of the lemma hold for each $\omega \in \mathcal{H}_{2}$. Hence, fix an arbitrary $\hat{\omega} \in \mathcal{H}_{2}$ for the remainder of the proof.

We first prove that $\varphi_{p, q}^{-1} \circ \varphi_{p, q}(t)=t$ for $t \in \Lambda$. Since the identities (8) and (12) are guaranteed by condition 1 , no point $t \in L^{-1}\left(\mathbb{R}_{+}\right)$is isolated from the right in $\Lambda$. Hence, since by condition $4, \varphi_{p, q}$ is strictly increasing on $\Lambda$, it follows that $\varphi_{p, q}^{-1} \circ \varphi_{p, q}(t)=t$ for $t \in L^{1}\left(\mathbb{R}_{+}\right)$. Next, note that by (12), which is guaranteed by condition 1 , it follows that if $t \in \Lambda \backslash L^{1}\left(\mathbb{R}_{+}\right)$, then $t=G_{i}$ for some $i \geq 1$. Hence, since by condition $5, V_{1}\left(Y,\left[G_{i}, D_{i} \wedge\left(G_{i}+2^{-j}\right)\right]\right)>0$ for $i, j \geq 1$, it follows since by condition $3, \varphi_{p, q}$ is well-defined and non-decreasing on $\mathbb{R}_{+}$, that using (39), $\varphi_{p, q}\left(t^{\prime}\right)>$ $\varphi_{p, q}(t)$ for $t^{\prime}>t$. Hence, it follows that $\varphi_{p, q}^{-1} \circ \varphi_{p, q}(t)=t$ for $t \in \Lambda \backslash L^{1}\left(\mathbb{R}_{+}\right)$, and so we have now shown that $\varphi_{p, q}^{-1} \circ \varphi_{p, q}(t)=t$ for $t \in \Lambda$, as desired.

Next, we show that $\varphi_{p, q}^{-1}$ is Hölder continuous on $\varphi_{p, q}(\Lambda)$ with Hölder exponent $1 / q$ and Hölder constant $H\left(\varphi_{p, q}^{-1}\right) \leq 1$. First note that since by condition $3, \varphi_{p, q}$ is well-defined on $\mathbb{R}_{+}$, it follows using the definition of $\varphi_{p, q}$ in (39) that

$$
\varphi_{p, q}(t)=V_{q}\left(L^{-1},[0, L(t)]\right), \quad t \in L^{-1}\left(\mathbb{R}_{+}\right) .
$$

Trivially, $L^{-1}=e \circ L^{-1}$, where $e: \mathbb{R}_{+} \mapsto \mathbb{R}_{+}$is the identity function, and so since $L^{-1}$ is non-decreasing by condition 2 , the change-of-variable formula for strong $q$-variation (see (P4) of Chistyakov and Galkin [7]) implies that

$$
\varphi_{p, q}(t)=V_{q}\left(L^{-1},[0, L(t)]\right)=V_{q}\left(e, L^{-1}([0, L(t)])\right)=V_{q}\left(e,\left(L^{-1}\left(\mathbb{R}_{+}\right)\right)_{t}^{-}\right), \quad t \geq 0,
$$

where the final equality follows since by condition $2, L^{-1}$ is non-decreasing, and since $L^{-1} \circ L(t)=t$ for $t \in L^{-1}\left(\mathbb{R}_{+}\right)$. Now note that since by condition $3, \varphi_{p, q}$ is continuous on $\mathbb{R}_{+}$with $\varphi_{p, q}\left(\mathbb{R}_{+}\right)=\mathbb{R}_{+}$, it follows that 
$\varphi_{p, q} \circ \varphi_{p, q}^{-1}(\tau)=\tau$ for $\tau \in \mathbb{R}_{+}$. Moreover, since it has been shown in the preceding discussion that $\varphi_{p, q}^{-1} \circ \varphi_{p, q}(t)=t$ for $t \in \Lambda$, it follows that $\varphi_{p, q}^{-1}(\tau) \in L^{-1}\left(\mathbb{R}_{+}\right)$for $\tau \in \varphi_{p, q}\left(L^{-1}\left(\mathbb{R}_{+}\right)\right)$. Thus, for $\tau, \tau^{\prime} \in \varphi_{p, q}\left(L^{-1}\left(\mathbb{R}_{+}\right)\right)$with $\tau \leq \tau^{\prime}$, by the minimality property and the semi-additivity property of strong $q$-variation (see (P1) and (P3) of Chistyakov and Galkin [7]), it follows that

$$
\begin{aligned}
\left|\varphi_{p, q}^{-1}\left(\tau^{\prime}\right)-\varphi_{p, q}^{-1}(\tau)\right|^{q} & \leq V_{q}\left(e,\left(L^{-1}\left(\mathbb{R}_{+}\right)\right)_{\varphi_{p, q}^{-1}(\tau)}^{\varphi_{p,}^{-1}\left(\tau^{\prime}\right)}\right) \\
& \leq V_{q}\left(e,\left(L^{-1}\left(\mathbb{R}_{+}\right)\right)_{\varphi_{p, q}^{-1}\left(\tau^{\prime}\right)}^{-}\right)-V_{q}\left(e,\left(L^{-1}\left(\mathbb{R}_{+}\right)\right)_{\varphi_{p, q}^{-1}(\tau)}^{-}\right) \\
& =\tau^{\prime}-\tau .
\end{aligned}
$$

Thus, $\varphi_{p, q}^{-1}$ meets the desired Hölder conditions on $\varphi_{p, q}\left(L^{-1}\left(\mathbb{R}_{+}\right)\right)$.

We now complete the proof by showing that $\varphi_{p, q}^{-1}$ meets the desired Hölder conditions on all of $\varphi_{p, q}(\Lambda)$. Let $\tau, \tau^{\prime} \in$ $\varphi_{p, q}(\Lambda)$. If $\tau, \tau^{\prime} \in \varphi_{p, q}\left(L^{-1}\left(\mathbb{R}_{+}\right)\right)$, then the Hölder conditions are met by the preceding paragraph. On the other hand, suppose that $\tau \in \varphi_{p, q}\left(L^{-1}\left(\mathbb{R}_{+}\right)\right)$and $\tau^{\prime} \in \varphi_{p, q}(\Lambda) \backslash \varphi_{p, q}\left(L^{-1}\left(\mathbb{R}_{+}\right)\right)$. Let $t \in L^{-1}\left(\mathbb{R}_{+}\right)$and $t^{\prime} \in \Lambda \backslash L^{-1}\left(\mathbb{R}_{+}\right)$be such that $\varphi_{p, q}(t)=\tau$ and $\varphi_{p, q}\left(t^{\prime}\right)=\tau^{\prime}$. By the identity (12), which holds by condition 1 , it follows that $t^{\prime}=G_{i}$ for some $i \geq 1$. Thus, using the fact by condition 1 that there are no isolated points in $\Lambda$ and that the identities (8) and (12) hold, it is straightforward to show that there exists a non-decreasing sequence $\left\{t_{k}^{\prime}\right\}$ of elements of $L^{-1}\left(\mathbb{R}_{+}\right)$such that $t_{k}^{\prime} \uparrow G_{i}$ and $L\left(t_{k}^{\prime}\right) \uparrow L\left(G_{i}\right)$. Hence, by the continuity of $\varphi_{p, q}$ on $\mathbb{R}_{+}$, which is guaranteed by condition 3 , it follows that $\tau_{k}^{\prime}=\varphi_{p, q}\left(t_{k}^{\prime}\right) \uparrow \varphi_{p, q}\left(G_{i}\right)=\tau^{\prime}$. Thus, since $\tau_{k}^{\prime} \in \varphi_{p, q}\left(L^{-1}\left(\mathbb{R}_{+}\right)\right)$, and noting that $\varphi_{p, q}^{-1} \circ \varphi_{p, q}(t)=t$ for $t \in \Lambda$ implies that $\varphi_{p, q}^{-1}\left(\tau_{k}^{\prime}\right)=t_{k}^{\prime} \uparrow t^{\prime}=\varphi_{p, q}^{-1}\left(\tau^{\prime}\right)$, it follows that

$$
\left|\varphi_{p, q}^{-1}(\tau)-\varphi_{p, q}^{-1}\left(\tau^{\prime}\right)\right|=\lim _{k \rightarrow \infty}\left|\varphi_{p, q}^{-1}(\tau)-\varphi_{p, q}^{-1}\left(\tau_{k}^{\prime}\right)\right| \leq \lim _{k \rightarrow \infty}\left|\tau-\tau_{k}^{\prime}\right|^{1 / q}=\left|\tau-\tau^{\prime}\right|^{1 / q} .
$$

In a similar manner, it may be shown that $\varphi_{p, q}^{-1}$ meets the desired Hölder conditions if $\tau, \tau^{\prime} \in \varphi_{p, q}(\Lambda) \backslash$ $\varphi_{p, q}\left(L^{-1}\left(\mathbb{R}_{+}\right)\right)$.

Now, for each $p>\alpha$ and $1>q>\alpha / 2$, let

$$
\Phi_{i}=\left(\frac{V_{1}\left(\varphi_{p, q},\left[G_{i}, D_{i}\right]\right)}{V_{p}\left(Y,\left[G_{i}, D_{i}\right]\right)}\right)^{1 / p}, \quad i \geq 1 .
$$

Lemma 5.7. For each $p>\alpha$ and $1>q>\alpha / 2$ and $i \geq 1$, it follows that $P^{0}$-a.s., $Y \circ \varphi_{p, q}^{-1} \circ \varphi_{p, q}(t)=Y(t)$ on $t \in\left[G_{i}, D_{i}\right]$. Moreover, $Y \circ \varphi_{p, q}^{-1}$ is $P^{0}$-a.s. Hölder continuous on $\left[\varphi_{p, q}\left(G_{i}\right), \varphi_{p, q}\left(D_{i}\right)\right]$ with Hölder exponent $1 / p$ and Hölder constant $H_{i}\left(Y \circ \varphi_{p, q}^{-1}\right) \leq 1 / \Phi_{i}$.

Proof. Let $p>\alpha$ and $1>q>\alpha / 2$ and $i \geq 1$. Since $\varphi_{p, q}: \mathbb{R}_{+} \mapsto \mathbb{R}_{+}$is, by Proposition 5.5, $P^{0}$-a.s. well-defined, it follows by (39) that $P^{0}$-a.s.,

$$
\frac{V_{1}\left(\varphi_{p, q},\left[G_{i}, D_{i}\right]\right)}{V_{p}\left(Y,\left[G_{i}, D_{i}\right]\right)} \cdot V_{p}\left(Y,\left[G_{i}, t\right]\right)=V_{p}\left(\Phi_{i} Y,\left[G_{i}, t\right]\right), \quad t \in\left[G_{i}, D_{i}\right],
$$

where $\Phi_{i}$ is defined in (44) above. Next, define the right-continuous inverse function $V_{p}^{-1}\left(\Phi_{i} Y, \cdot\right):\left[0, V_{p}\left(\Phi_{i} Y\right.\right.$, $\left.\left.\left[G_{i}, D_{i}\right]\right)\right] \mapsto\left[G_{i}, D_{i}\right]$ by

$$
V_{p}^{-1}\left(\Phi_{i} Y, \tau\right)=\inf \left\{t \in\left[G_{i}, D_{i}\right]: V_{p}\left(\Phi_{i} Y,\left[G_{i}, t\right]\right)>\tau\right\}, \quad \tau \in\left[0, V_{p}\left(\Phi_{i} Y,\left[G_{i}, D_{i}\right]\right)\right],
$$

where $\inf \varnothing=D_{i}$. Since $Y$ is $P^{0}$-a.s. continuous, it follows that $V_{p}\left(\Phi_{i} Y, \cdot\right)$ is $P^{0}$-a.s. continuous as well, and so $P^{0}$-a.s.,

$$
V_{p}\left(\Phi_{i} Y, V_{p}^{-1}\left(\Phi_{i} Y, \tau\right)\right)=\tau, \quad \tau \in\left[0, V_{p}\left(\Phi_{i} Y,\left[G_{i}, D_{i}\right]\right)\right]
$$


It then follows that $P^{0}$-a.s.,

$$
Y \circ V_{p}^{-1}\left(\Phi_{i} Y\right) \circ V_{p}\left(\Phi_{i} Y,\left[G_{i}, t\right]\right)=Y(t), \quad t \in\left[G_{i}, D_{i}\right],
$$

and, using the same argument as in the proof of Lemma 3.3 of [7], that $Y \circ V_{p}^{-1}\left(\Phi_{i} Y, \cdot\right):\left[0, V_{p}\left(\Phi_{i} Y,\left[G_{i}, D_{i}\right]\right)\right] \mapsto \mathbb{R}^{2}$ is $P^{0}$-a.s. Hölder continuous with Hölder exponent $1 / p$ and Hölder constant $H\left(Y \circ V_{p}^{-1}\left(\Phi_{i} Y, \cdot\right)\right) \leq 1 / \Phi_{i}$.

Now recall by (39) that $P^{0}$-a.s.,

$$
\varphi_{p, q}(t)=\varphi_{p, q}\left(G_{i}\right)+V_{p}\left(\Phi_{i} Y,\left[G_{i}, t\right]\right), \quad t \in\left[G_{i}, D_{i}\right] .
$$

Moreover, by (45), $P^{0}$-a.s.,

$$
V_{p}\left(\Phi_{i} Y,\left[G_{i}, D_{i}\right]\right)=V_{1}\left(\varphi_{p, q},\left[G_{i}, D_{i}\right]\right)=\varphi_{p, q}\left(D_{i}\right)-\varphi_{p, q}\left(G_{i}\right) .
$$

Hence, in order to complete the proof, it suffices by the preceding paragraph to show that $P^{0}$-a.s.,

$$
V_{p}^{-1}\left(\Phi_{i} Y, \tau\right)=\varphi_{p, q}^{-1}\left(\varphi_{p, q}\left(G_{i}\right)+\tau\right), \quad \tau \in\left[0, V_{p}\left(\Phi_{i} Y,\left[G_{i}, D_{i}\right]\right)\right]=\left[0, \varphi_{p, q}\left(D_{i}\right)-\varphi_{p, q}\left(G_{i}\right)\right] .
$$

However, the fact that by Proposition 5.5, $\varphi_{p, q}$ is $P^{0}$-a.s. non-decreasing, implies that $P^{0}$-a.s.,

$$
\begin{aligned}
\varphi_{p, q}^{-1}\left(\varphi_{p, q}\left(G_{i}\right)+\tau\right) & =\inf \left\{t \geq 0: \varphi_{p, q}(t)>\varphi_{p, q}\left(G_{i}\right)+\tau\right\} \\
& =\inf \left\{t \in\left[G_{i}, D_{i}\right]: \varphi_{p, q}(t)>\varphi_{p, q}\left(G_{i}\right)+\tau\right\},
\end{aligned}
$$

for $\tau \in\left[0, \varphi_{p, q}\left(D_{i}\right)-\varphi_{p, q}\left(G_{i}\right)\right]$. Then, by (46), $P^{0}$-a.s.,

$$
\begin{aligned}
\inf \left\{t \in\left[G_{i}, D_{i}\right]: \varphi_{p, q}(t)>\varphi_{p, q}\left(G_{i}\right)+\tau\right\} & =\inf \left\{t \in\left[G_{i}, D_{i}\right]: V_{p}\left(\Phi_{i} Y,\left[G_{i}, t\right]\right)>\tau\right\} \\
& =V_{p}^{-1}\left(\Phi_{i} Y, \tau\right),
\end{aligned}
$$

for $\tau \in\left[0, \varphi_{p, q}\left(D_{i}\right)-\varphi_{p, q}\left(G_{i}\right)\right]$.

The proof of Proposition 5.1 is as follows.

Proof of Proposition 5.1. Combining Lemmas 5.6 and 5.7 above, it follows that for each $p>\alpha$ and $1>q>\alpha / 2, P^{0}$ a.s., $Y \circ \varphi_{p, q}^{-1} \circ \varphi_{p, q}(t)=Y(t)$ for $t \in \mathbb{R}_{+}$. It is also immediate by Proposition 5.5 that $\varphi_{p, q}$ is $P^{0}$-a.s. non-decreasing on $\mathbb{R}_{+}$, with $\varphi_{p, q}\left(\mathbb{R}_{+}\right)=\mathbb{R}_{+}$. Hence, in order to complete the proof, it suffices by Theorem 3.1 of [7] to show that for each $\alpha<p<2$, there exists some $\alpha / 2<q<p / 2<1$ such that $Y \circ \varphi_{p, q}^{-1}$ is $P^{0}$-a.s. locally Hölder continuous on $\mathbb{R}_{+}$ with Hölder exponent $1 / p$.

Let $\mathcal{H}_{3}$ be the set of all $\omega \in C_{S}$ such that the following six conditions hold,

1. Identity (12) holds with $D_{i} \rightarrow+\infty$ as $i \rightarrow \infty$,

2. $\varphi_{p, q}: \mathbb{R}_{+} \mapsto \mathbb{R}_{+}$is well-defined, non-decreasing and continuous, with $\varphi_{p, q}\left(\mathbb{R}_{+}\right)=\mathbb{R}_{+}$,

3. $\varphi_{p, q}^{-1} \circ \varphi_{p, q}(t)=t$ for $t \in \Lambda$, and $\varphi_{p, q}^{-1}$ Hölder continuous on $\varphi_{p, q}(\Lambda)$ with Hölder exponent $1 / q$ and Hölder constant $H\left(\varphi_{p, q}^{-1}\right) \leq 1$,

4. For each $i \geq 1, Y \circ \varphi_{p, q}^{-1} \circ \varphi_{p, q}(t)=Y(t)$ on $t \in\left[G_{i}, D_{i}\right]$. Moreover, $Y \circ \varphi_{p, q}^{-1}$ is Hölder continuous on $\left[\varphi_{p, q}\left(G_{i}\right), \varphi_{p, q}\left(D_{i}\right)\right]$ with Hölder exponent $1 / p$ and Hölder constant $H_{i}\left(Y \circ \varphi_{p, q}^{-1}\right) \leq 1 / \Phi_{i}$,

5. $X: \mathbb{R}_{+} \mapsto \mathbb{R}^{2}$ is locally Hölder continuous with Hölder exponent $\eta$ for each $0 \leq \eta<1 / 2$,

6. The components of $R^{-1} Y$ are non-decreasing on $\left[G_{i}, D_{i}\right]$ for each $i \geq 1$.

By the discussion in Section 3, it is straightforward to show that condition 1 holds $P^{0}$-a.s. Proposition 5.5, and Lemmas 5.3 and 5.6 imply that conditions 2 through 4 hold $P^{0}$-a.s. By Part 1 of Theorem 4.2 of Section $4, X$ is a standard 2-dimensional Brownian motion under $P^{0}$, and so condition 5 holds $P^{0}$-a.s. by Remark 2.12 of [21]. Condition 6 holds $P^{0}$-a.s. by Part 2 of Theorem 4.2. Thus, $P^{0}\left(\mathcal{H}_{3}\right)=1$. In order to complete the proof, it therefore 
suffices to show that for each $\omega \in \mathcal{H}_{3}$, for each $\alpha<p<2$, there exists some $\alpha / 2<q<p / 2<1$ such that $Y$ 。 $\varphi_{p, q}^{-1}(\cdot, \omega)$ is locally Hölder continuous on $\mathbb{R}_{+}$with Hölder exponent $1 / p$. Hence, fix an arbitrary $\hat{\omega} \in \mathcal{H}_{3}$ for the remainder of the proof.

First note that since by condition $1, D_{i} \rightarrow+\infty$ as $i \rightarrow \infty$, and since by condition $2, \varphi_{p, q}(t) \rightarrow+\infty$ as $t \rightarrow+\infty$, it suffices to show that $Y \circ \varphi_{p, q}^{-1}$ is Hölder continuous on $\left[0, \varphi_{p, q}\left(D_{i}\right)\right]$ for each $i \geq 1$. Let $i \geq 1$ and $\tau, \tau^{\prime} \in\left[0, \varphi_{p, q}\left(D_{i}\right)\right]$ with $0 \leq \tau \leq \tau^{\prime} \leq \varphi_{p, q}\left(D_{i}\right)$ such that $\tau, \tau^{\prime} \in \varphi_{p, q}(\Lambda)$. Then, by condition $3, \varphi_{p, q}^{-1}(\tau), \varphi_{p, q}^{-1}\left(\tau^{\prime}\right) \in \Lambda$, and so $Y \circ$ $\varphi_{p, q}^{-1}(\tau)=-X \circ \varphi_{p, q}^{-1}(\tau)$ and $Y \circ \varphi_{p, q}^{-1}\left(\tau^{\prime}\right)=-X \circ \varphi_{p, q}^{-1}\left(\tau^{\prime}\right)$. This then implies that

$$
\left\|Y \circ \varphi_{p, q}^{-1}\left(\tau^{\prime}\right)-Y \circ \varphi_{p, q}^{-1}(\tau)\right\|=\left\|X \circ \varphi_{p, q}^{-1}\left(\tau^{\prime}\right)-X \circ \varphi_{p, q}^{-1}(\tau)\right\| .
$$

Now, by condition 5 , for each $0 \leq \eta<1 / 2$ and $T \geq 0$, there exists a constant $C_{\eta, T}$ such that

$$
\|X(t)-X(s)\| \leq C_{\eta, T}|t-s|^{\eta}, \quad 0 \leq s \leq t \leq T .
$$

Moreover, by condition 3 and the fact that $\tau, \tau^{\prime} \in\left[0, \varphi_{p, q}\left(D_{i}\right)\right]$ with $\tau, \tau^{\prime} \in \varphi_{p, q}(\Lambda)$, it follows since $\varphi_{p, q}: \mathbb{R}_{+} \mapsto \mathbb{R}_{+}$ is non-decreasing by condition 2 , that $0 \leq \varphi_{p, q}^{-1}(\tau) \leq \varphi_{p, q}^{-1}\left(\tau^{\prime}\right) \leq D_{i}$. Therefore, by (48), for each $0 \leq \eta<1 / 2$, it follows again by condition 3 that

$$
\left\|X \circ \varphi_{p, q}^{-1}\left(\tau^{\prime}\right)-X \circ \varphi_{p, q}^{-1}(\tau)\right\| \leq C_{\eta, D_{i}}\left|\varphi_{p, q}^{-1}\left(\tau^{\prime}\right)-\varphi_{p, q}^{-1}(\tau)\right|^{\eta} \leq C_{\eta, D_{i}}\left|\tau^{\prime}-\tau\right|^{\eta / q} .
$$

Now let $0 \leq \eta^{\star}<1 / 2$ be such that $q<\eta^{\star} p$. Note that such an $\eta^{\star}$ exists since by assumption $q<p / 2$. Then $\eta^{\star} / q>1 / p$, and since $0 \leq \tau \leq \tau^{\prime} \leq \varphi_{p, q}\left(D_{i}\right)$, it follows that $\left|\tau^{\prime}-\tau\right|^{\eta^{\star} / q} \leq K_{\eta^{\star}, i}\left|\tau^{\prime}-\tau\right|^{1 / p}$, where $K_{\eta^{\star}, i}=$ $\left(\varphi_{p, q}\left(D_{i}\right)\right)^{\eta^{\star} / q-1 / p}$. Hence, by (47) and (49),

$$
\left\|Y \circ \varphi_{p, q}^{-1}\left(\tau^{\prime}\right)-Y \circ \varphi_{p, q}^{-1}(\tau)\right\| \leq K_{\eta^{\star}, i} C_{\eta^{\star}, D_{i}}\left|\tau^{\prime}-\tau\right|^{1 / p} .
$$

Next, suppose that $\tau, \tau^{\prime} \in\left[0, \varphi_{p, q}\left(D_{i}\right)\right]$ with $0 \leq \tau \leq \tau^{\prime} \leq \varphi_{p, q}\left(D_{i}\right)$, and such that $\tau \in \varphi_{p, q}(\Lambda)$ and $\tau^{\prime} \notin \varphi_{p, q}(\Lambda)$. In this case, by conditions 1 and 2 , it follows that $\tau^{\prime} \in\left(\varphi_{p, q}\left(G_{j}\right), \varphi_{p, q}\left(D_{j}\right)\right)$ for some $j \geq 1$. By condition 4 , it follows that

$$
\left\|Y \circ \varphi_{p, q}^{-1}\left(\tau^{\prime}\right)-Y \circ \varphi_{p, q}^{-1}\left(\varphi_{p, q}\left(G_{j}\right)\right)\right\| \leq\left(1 / \Phi_{j}\right)\left|\tau^{\prime}-\varphi_{p, q}\left(G_{j}\right)\right|^{1 / p} .
$$

Also since $\varphi_{p, q}\left(G_{j}\right) \in \varphi_{p, q}(\Lambda) \cap\left[0, \varphi_{p, q}\left(D_{i}\right)\right]$, it follows by (50) that

$$
\left\|Y \circ \varphi_{p, q}^{-1}\left(\varphi_{p, q}\left(G_{j}\right)\right)-Y \circ \varphi_{p, q}^{-1}(\tau)\right\| \leq K_{\eta^{\star}, i} C_{\eta^{\star}, D_{i}}\left|\varphi_{p, q}\left(G_{j}\right)-\tau\right|^{1 / p} .
$$

Hence, since $0 \leq \tau \leq \varphi_{p, q}\left(G_{j}\right)<\tau^{\prime} \leq \varphi_{p, q}\left(D_{i}\right)$, it follows by the triangle inequality that

$$
\left\|Y \circ \varphi_{p, q}^{-1}\left(\tau^{\prime}\right)-Y \circ \varphi_{p, q}^{-1}(\tau)\right\| \leq\left(\left(1 / \Phi_{j}\right)+K_{\eta^{\star}, i} C_{\eta^{\star}, D_{i}}\right)\left|\tau^{\prime}-\tau\right|^{1 / p} .
$$

Similar reasoning leads to the same inequality if we assume that $\tau, \tau^{\prime} \in\left[0, \varphi_{p, q}\left(D_{i}\right)\right]$ with $0 \leq \tau \leq \tau^{\prime} \leq \varphi_{p, q}\left(D_{i}\right)$, and such that $\tau \notin \varphi_{p, q}(\Lambda)$ and $\tau^{\prime} \in \varphi_{p, q}(\Lambda)$.

Next consider $\tau, \tau^{\prime} \in\left[0, \varphi_{p, q}\left(D_{i}\right)\right]$ with $0 \leq \tau \leq \tau^{\prime} \leq \varphi_{p, q}\left(D_{i}\right)$, and such that $\tau, \tau^{\prime} \notin \varphi_{p, q}(\Lambda)$. Suppose first that $\tau \in\left(\varphi_{p, q}\left(G_{j}\right), \varphi_{p, q}\left(D_{j}\right)\right)$ and $\tau \in\left(\varphi_{p, q}\left(G_{k}\right), \varphi_{p, q}\left(D_{k}\right)\right)$ with $j \neq k$. In this case, similar reasoning to the above leads to the bound

$$
\left\|Y \circ \varphi_{p, q}^{-1}\left(\tau^{\prime}\right)-Y \circ \varphi_{p, q}^{-1}(\tau)\right\| \leq\left(\left(1 / \Phi_{j}\right)+K_{\eta^{\star}, i} C_{\eta^{\star}, D_{i}}+\left(1 / \Phi_{k}\right)\right)\left|\tau^{\prime}-\tau\right|^{1 / p} .
$$

On the other hand, if $\tau, \tau^{\prime} \in\left(\varphi_{p, q}\left(G_{j}\right), \varphi_{p, q}\left(D_{j}\right)\right)$, it is immediate by condition 4 that

$$
\left\|Y \circ \varphi_{p, q}^{-1}\left(\tau^{\prime}\right)-Y \circ \varphi_{p, q}^{-1}\left(\varphi_{p, q}(\tau)\right)\right\| \leq\left(1 / \Phi_{j}\right)\left|\tau^{\prime}-\tau\right|^{1 / p} .
$$

Combining the cases (50), (51), (52) and (53) we now obtain that

$$
\left\|Y \circ \varphi_{p, q}^{-1}\left(\tau^{\prime}\right)-Y \circ \varphi_{p, q}^{-1}(\tau)\right\| \leq\left(2 \Theta_{i}+K_{\eta^{\star}, i} C_{\eta^{\star}, D_{i}}\right)\left|\tau^{\prime}-\tau\right|^{1 / p} \quad \text { for } \tau, \tau^{\prime} \in\left[0, \varphi_{p, q}\left(D_{i}\right)\right],
$$


where

$$
\Theta_{i}=\sup \left\{\left(1 / \Phi_{k}\right): D_{k} \leq D_{i}\right\} .
$$

Hence, in order to show that $Y \circ \varphi_{p, q}^{-1}$ is Hölder continuous on $\left[0, \varphi_{p, q}\left(D_{i}\right)\right]$ with Hölder exponent $1 / p$, it suffices to show that $\Theta_{i}<+\infty$. Using the definition of $\Phi_{i}$ in (44) it suffices to show that there exists $0<K_{i}<+\infty$ such that for $k \geq 1$,

$$
1\left\{D_{k} \leq D_{i}\right\}\left(\frac{V_{p}\left(Y,\left[G_{k}, D_{k}\right]\right)}{V_{1}\left(\varphi_{p, q},\left[G_{k}, D_{k}\right]\right)}\right)^{1 / p}<K_{i} .
$$

Recall the definition of the reflection matrix $R$ from Section 4.2 and let $\bar{Y}=R^{-1} Y$. By condition 6, the components of $\bar{Y}$ are non-decreasing on $\left[G_{k}, D_{k}\right]$ for each $k \geq 1$. Thus, since $Z=X+Y$ and recalling that $Z\left(G_{k}\right)=Z\left(D_{k}\right)=0$, it follows that

$$
Y\left(D_{k}\right)-Y\left(G_{k}\right)=-\left(X\left(D_{k}\right)-X\left(G_{k}\right)\right) .
$$

On the other hand, by the definition of $\bar{Y}$,

$$
\bar{Y}\left(D_{k}\right)-\bar{Y}\left(G_{k}\right)=R^{-1}\left(Y\left(D_{k}\right)-Y\left(G_{k}\right)\right) .
$$

Hence, using the local Hölder continuity (48) of $X$ given by condition 5, it follows that for $k \geq 1$ such that $D_{k} \leq D_{i}$,

$$
\left\|\bar{Y}\left(D_{k}\right)-\bar{Y}\left(G_{k}\right)\right\| \leq\left\|R^{-1}\right\|\left\|X\left(D_{k}\right)-X\left(G_{k}\right)\right\| \leq\left\|R^{-1}\right\| C_{\eta, D_{i}}\left(D_{i}-G_{i}\right)^{\eta},
$$

for each $0 \leq \eta<1 / 2$, where $\left\|R^{-1}\right\|$ denotes the operator norm of $R^{-1}$. Next, for $x=\left(x_{1}, x_{2}\right) \in \mathbb{R}^{2}$, it is straightforward to show that

$$
\|x\|^{\rho} \leq\left|x_{1}\right|^{\rho}+\left|x_{2}\right|^{\rho} \quad \text { for } 0 \leq \rho \leq 2 .
$$

Hence, letting $\bar{Y}=\left(\bar{Y}_{1}, \bar{Y}_{2}\right)$ and using the definition of strong $p$-variation, it is immediate since $\alpha<p<2$ that

$$
V_{p}\left(\bar{Y},\left[G_{k}, D_{k}\right]\right) \leq V_{p}\left(\bar{Y}_{1},\left[G_{k}, D_{k}\right]\right)+V_{p}\left(\bar{Y}_{2},\left[G_{k}, D_{k}\right]\right) .
$$

However, since $\bar{Y}$ has components which are non-decreasing on $\left[G_{k}, D_{k}\right]$ and $1<\alpha<p$, it follows that

$$
V_{p}\left(\bar{Y}_{\ell},\left[G_{k}, D_{k}\right]\right)=\left(\bar{Y}_{\ell}\left(D_{k}\right)-\bar{Y}_{\ell}\left(G_{k}\right)\right)^{p} \leq\left\|\bar{Y}\left(D_{k}\right)-\bar{Y}\left(G_{k}\right)\right\|^{p} \quad \text { for } \ell=1,2 .
$$

Thus, by (55),

$$
V_{p}\left(\bar{Y},\left[G_{k}, D_{k}\right]\right) \leq \bar{C}_{\eta, D_{i}}\left(D_{k}-G_{k}\right)^{\eta p}
$$

where $\bar{C}_{\eta, D_{i}}=2\left(\left\|R^{-1}\right\| C_{\eta, D_{i}}\right)^{p}$. Next, note that

$$
V_{p}\left(Y,\left[G_{k}, D_{k}\right]\right)=V_{p}\left(R \bar{Y},\left[G_{k}, D_{k}\right]\right) \leq\|R\|^{p} V_{p}\left(\bar{Y},\left[G_{k}, D_{k}\right]\right),
$$

where $\|R\|$ denotes the operator norm of $R$. Hence,

$$
V_{p}\left(Y,\left[G_{k}, D_{k}\right]\right) \leq \overline{\bar{C}}_{\eta, D_{i}}\left(D_{k}-G_{k}\right)^{\eta p},
$$

where $\overline{\bar{C}}_{\eta, D_{i}}=2\left(\|R\|\left\|R^{-1}\right\| C_{\eta, D_{i}}\right)^{p}$. Now again let $0 \leq \eta^{\star}<1 / 2$ be such that $q<\eta^{\star} p$. Then, since $\left(D_{k}-G_{k}\right)<D_{i}$, it follows that

$$
\frac{V_{p}\left(Y,\left[G_{k}, D_{k}\right]\right)}{\left(D_{k}-G_{k}\right)^{q}} \leq \overline{\bar{C}}_{\eta^{\star}, D_{i}}
$$

where $\overline{\overline{\bar{C}}}_{\eta, D_{i}}=\left(D_{i}\right)^{\eta p-q} \overline{\bar{C}}_{\eta, D_{i}}$. Hence, (54) holds with $K_{i}=\left(\overline{\overline{\bar{C}}}_{\eta^{\star}, D_{i}}\right)^{1 / p}$. 


\section{Proof of Theorem 2.6}

Theorem 2.4 implies that $Z$ has the unique decomposition $Z=X+Y$, where for each $z \in S, X$ is a standard 2dimensional Brownian motion started at $z$ and $Y$ is a process of zero energy on $\left(C_{S}, \mathcal{F}, \mathcal{F}_{t}, P^{z}\right)$. In the proof of Theorem 2.4, it was also shown (see (37) and the ensuing arguments) that for each $2>p>\alpha$ and $z \in S$,

$$
P^{z}\left(V_{p}(Y,[0, T])<\infty\right)=1, \quad T \geq 0 .
$$

The fact that (56) may be extended to all $p>\alpha$ is an immediate consequence of the identity $V_{q}(Y,[0, T]) \leq$ $\left(V_{p}(Y,[0, T])\right)^{q / p}$ for $1 \leq p \leq q$ (see Remark 2.5 of Chistyakov and Galkin [7]). Hence, in order prove Theorem 2.6 it only remains to prove the partial converse result (5).

Recall from Section 3 the definition of $L$ as the local time of $Z$ at the origin and let $Y \circ L^{-1}$ be the process defined by $Y \circ L^{-1}=\left\{Y \circ L^{-1}(t), t \geq 0\right\}$. For each $t \geq 0$, we have by (10) that $L^{-1}(t)$ is a stopping time relative to the filtration $\mathcal{F}_{t}$ and so we may define the new filtration $\mathcal{F}_{L^{-1}}=\left\{\mathcal{F}_{L^{-1}(t)}, t \geq 0\right\}$.

Proposition 6.1. For each $z \in S$, the process $Y \circ L^{-1}$ is a Lévy process on $\left(C_{S}, \mathcal{F}, \mathcal{F}_{L^{-1}}, P^{z}\right)$. In particular, it is an $\alpha$-stable process on $\left(C_{S}, \mathcal{F}, \mathcal{F}_{L^{-1}}, P^{0}\right)$, i.e. under $P^{0}$,

$$
\lambda^{-\frac{1}{\alpha}} Y \circ L^{-1}(\lambda \cdot) \stackrel{\mathrm{d}}{=} Y \circ L^{-1}(\cdot), \quad \lambda>0 .
$$

Proof. Recall the notational remark from the end of Section 3. Let $0 \leq a<b$ be arbitrary. Then applying (13) to $s=L^{-1}(a)$, we obtain

$$
\begin{aligned}
L^{-1}(b) & =\inf \left\{t \geq L^{-1}(a): L(t)>b\right\} \\
& =\inf \left\{t \geq L^{-1}(a): a+L\left(t-L^{-1}(a), Z\left(L^{-1}(a)+\cdot\right)\right)>b\right\} \\
& =L^{-1}(a)+\inf \left\{u \geq 0: L\left(u, Z\left(L^{-1}(a)+\cdot\right)\right)>b-a\right\} \\
& =L^{-1}(a)+L^{-1}\left(b-a, Z\left(L^{-1}(a)+\cdot\right)\right),
\end{aligned}
$$

$P^{z}$-a.s., for every $z \in S$. Then, for any $B \in \mathcal{B}(S)$, by (26), (71), (58), (29), the Strong Markov Property (see Theorem 3.14 of Varadhan and Williams [28]), and the fact that by (12) $Z\left(L^{-1}(a)\right)=0$, we have

$$
\begin{aligned}
P^{z} & \left(Y\left(L^{-1}(b)\right)-Y\left(L^{-1}(a)\right) \in B \mid \mathcal{F}_{L^{-1}(a)}\right) \\
& =P^{z}\left(X\left(L^{-1}(a)\right)-X\left(L^{-1}(b)\right) \in B \mid \mathcal{F}_{L^{-1}(a)}\right) \\
& =P^{z}\left(F_{\gamma^{z}}(Z(\cdot))\left(L^{-1}(a)\right)-F_{\gamma^{z}}(Z(\cdot))\left(L^{-1}(b)\right) \in B \mid \mathcal{F}_{L^{-1}(a)}\right) \\
& =P^{z}\left(-F_{\gamma^{z}}\left(Z\left(L^{-1}(a)+\cdot\right)\right)\left(L^{-1}(b)-L^{-1}(a)\right) \in B \mid \mathcal{F}_{L^{-1}(a)}\right) \\
& =P^{z}\left(-F_{\gamma^{z}}\left(Z\left(L^{-1}(a)+\cdot\right)\right)\left(L^{-1}\left(b-a, Z\left(L^{-1}(a)+\cdot\right)\right)\right) \in B \mid \mathcal{F}_{L^{-1}(a)}\right) \\
& =P^{0}\left(-F_{\gamma^{z}}(Z(\cdot))\left(L^{-1}(b-a)\right) \in B\right) \\
& =P^{0}\left(-X\left(L^{-1}(b-a)\right) \in B\right)=P^{0}\left(Y\left(L^{-1}(b-a)\right) \in B\right),
\end{aligned}
$$

which shows that $Y \circ L^{-1}$ is indeed a Lévy process on $\left(C_{S}, \mathcal{F}, \mathcal{F}_{L^{-1}}, P^{z}\right)$. Next, we show that it is also an $\alpha$-stable process on $\left(C_{S}, \mathcal{F}, \mathcal{F}_{L^{-1}}, P^{0}\right)$.

Recall the function $\phi: S \mapsto \mathbb{R}$ from [31] given by $\phi(r, \theta)=r^{\alpha} \cos \left(\alpha \theta-\theta_{1}\right), r \geq 0,0 \leq \theta \leq \xi$ ( $r$ and $\theta$ are polar coordinates). It is shown in [31] that $\{\phi(Z(t)), t \geq 0\}$ is a local submartingale on $\left(C_{S}, \mathcal{F}, \mathcal{F}_{t}, P^{z}\right)$ for each $z \in S$, that can be uniquely decomposed as

$$
\phi(Z(t))=\phi(Z(0))+M(t)+A(t)
$$


where $M$ is a continuous local martingale, and $A$ is an adapted, measurable, non-decreasing, continuous process, and $M(0)=A(0)=0, P^{z}$-a.s. (see (2.6) in [31]). It is also shown in [31] that the processes $A$ and $M$ can be selected so that they do not depend on $z$. Moreover, by Theorem 2.6 in [31] we have that

$$
L(t)=c A(t), \quad t \geq 0, P^{z} \text {-a.s. }
$$

for some positive constant $c$. Next for all $\lambda>0$ we introduce the transformed processes

$$
\begin{aligned}
A_{\lambda}(t) & =\lambda^{-\alpha / 2} A(\lambda t), & X_{\lambda}(t) & =\lambda^{-1 / 2} X(\lambda t), \\
M_{\lambda}(t) & =\lambda^{-\alpha / 2} M(\lambda t), & Z_{\lambda}(t) & =\lambda^{-1 / 2} Z(\lambda t), \quad t \geq 0 .
\end{aligned}
$$

By Lemma 2.1 in [31], we have that $Z_{\lambda}(\cdot) \stackrel{\mathrm{d}}{=} Z(\cdot)$ under $P^{0}$. Consider now the following identities given in the proof of Theorem 2.6 of [31]:

$$
\begin{gathered}
\phi(Z(\cdot)) \stackrel{\mathrm{d}}{=} \phi\left(Z_{\lambda}(\cdot)\right)=\phi\left(\lambda^{-1 / 2} Z(\lambda \cdot)\right)=\lambda^{-\frac{\alpha}{2}} \phi(Z(\lambda \cdot)) \\
=\lambda^{-\frac{\alpha}{2}}(M(\lambda \cdot)+A(\lambda \cdot))=M_{\lambda}(\cdot)+A_{\lambda}(\cdot),
\end{gathered}
$$

where the identity in law is understood to be under $P^{0}$. By the uniqueness of the decomposition in (59), $A_{\lambda}(\cdot)$ must depend on $Z_{\lambda}(\cdot)$ in the same way as $A(\cdot)$ depends on $Z(\cdot)$. Since $A(\cdot)=A(\cdot) \circ Z(\cdot)$, so we have $P^{0}$-almost surely $A_{\lambda}(\cdot)=A \circ Z_{\lambda}(\cdot)$. Furthermore, $\left\{\gamma^{0}(n), n \geq 1\right\} \in \Gamma$ implies $\left\{\lambda^{-1 / 2} \gamma^{0}(n), n \geq 1\right\} \in \Gamma$, thus by Lemma 4.11 there exists a sequence $\left\{\epsilon^{\lambda}(n), n \geq 1\right\} \subset\left\{\gamma^{0}(n), n \geq 1\right\}$ such that

$$
F_{\lambda^{-1 / 2} \epsilon^{\lambda}}(\omega)=W(\cdot, \omega)=X(\cdot, \omega), \quad P^{0} \text {-a.s. }
$$

By (73), we have that $P^{0}$-almost surely

$$
X_{\lambda}(t)=\lambda^{-1 / 2} X(\lambda t)=\lambda^{-1 / 2} F_{\epsilon^{\lambda}}(Z(\cdot))(\lambda t)=F_{\lambda^{-1 / 2} \epsilon^{\lambda}}\left(\lambda^{-1 / 2} Z(\lambda \cdot)\right)(t)=F_{\lambda^{-1 / 2} \epsilon^{\lambda}}\left(Z_{\lambda}(\cdot)\right)(t) .
$$

We summarize all these as

$$
\left(X_{\lambda}(\cdot), A_{\lambda}(\cdot)\right)=\left(F_{\lambda^{-1 / 2} \epsilon^{\lambda}} \circ Z_{\lambda}(\cdot), A(\cdot) \circ Z_{\lambda}(\cdot)\right) \stackrel{\mathrm{d}}{=}\left(F_{\lambda^{-1 / 2}} \epsilon^{\lambda} \circ Z(\cdot), A(\cdot) \circ Z(\cdot)\right)=(X(\cdot), A(\cdot))
$$

under $P^{0}$, that is,

$$
\left(\lambda^{-\frac{1}{2}} X(\lambda \cdot), \lambda^{-\frac{\alpha}{2}} A(\lambda \cdot)\right) \stackrel{\mathrm{d}}{=}(X(\cdot), A(\cdot)) .
$$

However, the identity (60) implies

$$
\lambda^{-\frac{\alpha}{2}} L(\lambda t)=c \lambda^{-\frac{\alpha}{2}} A(\lambda t),
$$

and thus we have that

$$
\left(\lambda^{-\frac{1}{2}} X(\lambda \cdot), \lambda^{-\frac{\alpha}{2}} L(\lambda \cdot)\right) \stackrel{\mathrm{d}}{=}(X(\cdot), L(\cdot))
$$

We now conclude that

$$
\lambda^{-\frac{1}{2}} X\left(L^{-1}\left(\lambda^{\frac{\alpha}{2}} \cdot\right)\right) \stackrel{\mathrm{d}}{=} X\left(L^{-1}(\cdot)\right),
$$

which implies (57).

We now present the proof of Theorem 2.6. 
Proof of Theorem 2.6. Following the discussion at the outset of the section, it only remains to prove the identity (5). Let $T \geq 0$. Since $Y=-X$ on $\left(L^{-1}\left(\mathbb{R}_{+}\right)\right)_{T}^{-} \subset \Lambda_{T}^{-} \subset[0, T]$, it follows by Definition 2.5 that

$$
V_{p}(Y,[0, T]) \geq V_{p}\left(Y,\left(L^{-1}\left(\mathbb{R}_{+}\right)\right)_{T}^{-}\right)=V_{p}\left(X,\left(L^{-1}\left(\mathbb{R}_{+}\right)\right)_{T}^{-}\right), \quad p \geq 0 .
$$

Hence it suffices to show that $P^{0}\left(V_{p}\left(X,\left(L^{-1}\left(\mathbb{R}_{+}\right)\right)_{T}^{-}\right)<+\infty\right)=0$ for $0<p \leq \alpha$. For each $A>0$, let $T_{A}$ be defined by $L^{-1}(A)=T_{A}$. Since $L^{-1}$ is $P^{0}$-a.s. right-continuous with $L^{-1}(0)=0$, it follows that $T_{A} \Rightarrow 0$ as $A \downarrow 0$. Also recall that $P^{0}\left(T_{A}>0\right)=1$ for each $A>0$. Moreover, since $L^{-1}$ is $P^{0}$-a.s. non-decreasing, it follows by the change-ofvariables for $p$-variation (see (P4) of Chistyakov and Galkin [7]) that $V_{p}\left(X,\left(L^{-1}\left(\mathbb{R}_{+}\right)\right)_{T_{A}}^{-}\right)=V_{p}\left(X \circ L^{-1},[0, A]\right)$, $A>0$. Thus, in order to complete the proof it suffices to show that for $0<p \leq \alpha$,

$$
P^{0}\left(V_{p}\left(X \circ L^{-1},[0, A]\right)<+\infty\right)=0, \quad A>0 .
$$

Let $v$ be the Levy measure of $X \circ L^{-1}$ under $P^{0}$. According to Bretagnolle [3] (see also Simon [27]), (61) holds if and only if

$$
\int_{|u|<1}|u|^{p} v(d u)=\infty .
$$

However, since by Proposition 6.1, $Y \circ L^{-1}=-X \circ L^{-1}$ is an $\alpha$-stable process on $\left(C_{S}, \mathcal{F}, \mathcal{F}_{L^{-1}}, P^{0}\right)$, it follows by Theorem 14.3(ii) of [26] that $v(d B)=\int_{C} \int_{0}^{\infty} 1_{B}(r \xi) r^{-1-\alpha} d r \lambda(d \xi)$, where $C$ is the unit circle in $\mathbb{R}^{2}$ and $\lambda$ is a finite measure on $C$. (62) now follows immediately.

\section{Proof of Theorem 2.8}

Before providing the proof of Theorem 2.8, we first proceed with some propositions, starting with a result that condition (7) is necessary for the existence of a solution to the ESP.

Proposition 7.1. Let $z \in S$. If the $\operatorname{ESP}(S, d(\cdot))$ for the Brownian motion $X$ on $\left(C_{S}, \mathcal{F}, \mathcal{F}_{t}, P^{z}\right)$ has a solution $P^{z}$-a.s., then (7) holds.

Proof. Let the polar coordinates of $v_{i}$ be $\left(\left\|v_{i}\right\|, \beta_{i}\right)$ for $i=1,2$. Then $\beta_{1}=\theta_{1}+\pi / 2$ and $\beta_{2}=-\left(\pi / 2-\xi+\theta_{2}\right)$. Thus, since by assumption $1<\alpha<2$, it follows that $\xi<\beta_{1}<\pi$ and $-(\pi-\xi)<\beta_{2}<0$. In addition, the condition $\alpha>1$ implies that the angle of $v_{1}$ and $v_{2}$ on the side which contains $S$ is $\beta_{1}-\beta_{2}>\pi$. Let $C=\overline{\operatorname{co}}\left(V \cup\left\{\alpha v_{1}, \alpha \geq\right.\right.$ $\left.0\} \cup\left\{\alpha v_{2}, \alpha \geq 0\right\}\right)$, which is a closed convex cone, and assume that $C \neq \mathbb{R}^{2}$, which implies $C \cap S=\{0\}$. Let $\tilde{Z}, \tilde{Y}$ be a solution of the $\operatorname{ESP}(S, d(\cdot))$ for $X$. Let $B$ be an arbitrary open set in $C$ such that $0 \notin B$, and let $t>0$ arbitrary. Now we have $P^{z}(X(t) \in B)>0$, and also $X(t)+\tilde{Y}(t)=\tilde{Z}(t) \in S$. However, whenever $X(t) \in B$, then $\tilde{Y}(t) \in C$ implies $X(t)+\tilde{Y}(t) \in C$, which implies $X(t)+\tilde{Y}(t)=0$. But this is impossible, since a non-zero vector and its negative can not be in a closed convex cone that is neither $\mathbb{R}^{2}$, nor a single line.

We now proceed to show that if the condition (7) holds, then $(Z, Y)$ solves the $\operatorname{ESP}(S, d(\cdot))$ for $X$. We first provide some prepatory lemmas. For each $i=1,2$, let

$$
T_{\partial S^{i}}=\inf \left\{t>0: Z(t) \in \partial S^{i} \backslash\{0\}\right\} .
$$

Lemma 7.2. For each $i=1,2, P^{0}\left(T_{\partial S^{i}}>0\right)=0$.

Proof. Let $i=1$ or 2 , and

$$
T_{\partial S^{i}}^{c}=\inf \left\{t \geq 0: \frac{Z(c t)}{\sqrt{c}} \in \partial S^{i} \backslash\{0\}\right\}, \quad c>0 .
$$


Then, by Lemma 2.1 in [31],

$$
P^{0}\left(T_{\partial S^{i}}^{c}>t\right)=P^{0}\left(T_{\partial S^{i}}^{1}>t\right), \quad t \geq 0 .
$$

On the other hand,

$$
T_{\partial S^{i}}^{c}=\inf \left\{t \geq 0: Z(c t) \in \partial S^{i} \backslash\{0\}\right\}=\inf \left\{\frac{u}{c} \geq 0: Z(u) \in \partial S^{i} \backslash\{0\}\right\}=\frac{1}{c} T_{\partial S^{i}}^{1} .
$$

Hence,

$$
P^{0}\left(T_{\partial S^{i}}^{1}>c t\right)=P^{0}\left(T_{\partial S^{i}}^{1}>t\right)
$$

which implies $P^{0}\left(T_{\partial S^{i}}=0\right)+P^{0}\left(T_{\partial S^{i}}=\infty\right)=1$. By the Blumenthal 0-1 law, $P^{0}\left(T_{\partial S^{i}}=0\right)$ is either 0 or 1 . Hence, in order to complete the proof it suffices that $P^{0}\left(T_{\partial S^{i}}=\infty\right)<1$, and this is what we shall do.

By Lemma 4.2 in [29], for Lebesgue-almost every $t \in(0, \infty)$,

$$
P^{0}(Z(t) \in \partial S)=0
$$

However, by Lemma 2.1 in [31], for every $c>0$

$$
P^{0}(Z(t) \in \partial S)=P^{0}\left(\frac{Z(c t)}{\sqrt{c}} \in \partial S\right)=P^{0}(Z(c t) \in \partial S),
$$

and so (63) holds for every $t>0$. Now let $t>0$ be arbitrary and

$$
T_{\partial S^{i}}(t)=\inf \left\{s \geq t: Z(s) \in \partial S^{i} \backslash\{0\}\right\} .
$$

Then, by the Markov property,

$$
P^{0}\left(T_{\partial S^{i}}(t)<\infty\right)=E^{0}\left[P^{0}\left(T_{\partial S^{i}}(t)<\infty \mid Z(t)\right)\right]=E^{0}\left[P^{Z(t)}\left(T_{\partial S^{i}}<\infty\right)\right] .
$$

Let $T_{\partial S}=\inf \{t \geq 0: Z(t) \in \partial S\}$. By Proposition 4.9, for each $z \in \operatorname{int}(S)$ the law of $Z\left(\cdot \wedge T_{\partial S}\right)$ under $P^{z}$ coincides with the law of a Brownian motion started at $z$ and stopped at the first hitting time of $\partial S$. It follows that for all $z \in \operatorname{int}(S)$ we have $P^{z}\left(T_{\partial S}<\infty\right)=1$ and $P^{z}\left(Z\left(T_{\partial S}\right) \in \partial S^{i} \backslash\{0\}\right)>0$, which implies $P^{z}\left(T_{\partial S^{i}}<\infty\right)>0$. Then it follows from (63) that the last expression in (64) is strictly positive, and so also is the first expression there, i.e. $P^{0}\left(T_{\partial S^{i}}(t)<\infty\right)>0$. Thus, $P^{0}\left(T_{\partial S^{i}}<\infty\right)>0$.

Lemma 7.3. For each $z \in S$ and $P^{z}$-a.e. $\omega \in C_{s}$, the following holds: for the endpoint $D$ of each excursion interval of $Z(\omega)$ away from zero and each $\epsilon>0$, there exist $u_{1}, u_{2}, u \in(D, D+\epsilon)$ such that

$$
Z\left(u_{1}\right) \in \partial S^{1} \backslash\{0\}, \quad Z\left(u_{2}\right) \in \partial S^{2} \backslash\{0\}, \quad \text { and } \quad Z(u)=0 .
$$

Proof. Recall the sequence of stopping times $\left\{T_{k}^{n}, k \geq 1\right\}$ from the proof of Proposition 4.13, that is the sequence of endpoints of excursions away from zero with length larger than $1 / n$. By the Strong Markov Property, Proposition 7.2, and the regularity of the vertex (see Lemma 2.2 of [31]), for every $k, n \geq 1$, we have that $P^{z}$-almost surely there exist $u_{1}, u_{2}, u \in\left(T_{k}^{n}, T_{k}^{n}+\epsilon\right)$ such that (65) holds. Since the set of excursions is countable, and $\bigcup_{n \geq 1}\left\{T_{k}^{n}, k \geq 1\right\}$ is exactly the set of endpoints of all excursion intervals, the statement follows.

The following is an immediate corollary of Lemma 7.3.

Corollary 7.4. For each $z \in S$ and $P^{z}$-a.e. $\omega \in C_{S}$, the set $\Lambda$ has no isolated point.

We now present the proof that under (7), $(Z, Y)$ solves the $\operatorname{ESP}(S, d(\cdot))$ for $X$. 
Proposition 7.5. If (7) holds, then for each $z \in S,(Z, Y) P^{z}$-a.s. solves the ESP $(S, d(\cdot))$ for the Brownian motion $X$ on $\left(C_{S}, \mathcal{F}, \mathcal{F}_{t}, P^{z}\right)$.

Proof. Let $z \in S$. We will verify that $P^{z}$-a.s. $(Z, Y)$ satisfies Conditions 1 through 4 of Definition 2.7 with $\psi=X$ and $(\phi, \eta)=(Z, Y)$. Condition 1 is clear since by the Doob-Meyer type decomposition of Theorem 2.4, we have that $Z=X+Y$. Next, Condition 2 is immediate as well since $Z \in C_{S}$. Now recall that $X$ is a Brownian motion started at $z$ on $\left(C_{S}, \mathcal{F}, \mathcal{F}_{t}, P^{z}\right)$ and so $X$ is $P^{z}$-a.s continuous. Hence $Y=Z-X$ is $P^{z}$-a.s. continuous as well and so Condition 4 holds since $0 \in \overline{\mathrm{co}}[d(\phi(t))]$ for $t \geq 0$.

In order to complete the proof, it suffices to show that $P^{z}$-a.s. Condition 3 holds. That is, it suffices to show that $P^{z}$-a.s. for each $t \geq 0$ and $s \in[0, t]$,

$$
Y(t)-Y(s) \in \overline{\mathrm{co}}\left[\bigcup_{u \in(s, t]} d(Z(u))\right] .
$$

Let $0 \leq s<t$ arbitrary. We distinguish between two cases. In the first case, the interval $[s, t]$ lies entirely within a single excursion of $Z$ away from zero, i.e. $(s, t) \cap \Lambda=\varnothing$. In this case, by Part 2 of Theorem 4.2, $Y(v)=R U(v)$ where $U$ is non-decreasing on $[s, t]$, and $U_{i}$ is increasing on $[s, t]$ only at times $v \in[s, t]$ when $Z(v) \in \partial S^{i}$ for $i=i, 2$, and then (66) follows. Next, we show that (66) holds in the second case, where $(s, t) \cap \Lambda \neq \varnothing$. In this case, let $v \in(s, t) \cap \Lambda$. By Corollary 7.4, there exists another point $u$ besides $v$ in $(s, t) \cap \Lambda$, and, by Condition 3 of Definition 2.1, there exists an excursion interval included in $[u, v]$ or in $[v, u]$, depending on whether $u<v$ or $v<u$. In either case, by Lemma 7.3 this implies the existence of $u_{1}, u_{2} \in(s, t)$ such that $Z\left(u_{i}\right) \in \partial S^{i} \backslash\{0\}, i=1,2$. Then, by (7),

$$
\overline{\mathrm{co}}\left(\bigcup_{u \in(s, t]} d(Z(u))\right)=\overline{\operatorname{co}}\left(V \cup\left\{a v_{1}, a \geq 0\right\} \cup\left\{a v_{2}, a \geq 0\right\}\right)=\mathbb{R}^{2},
$$

and thus (66) is trivially satisfied.

The proof of Theorem 2.8 is now immediate.

Proof of Theorem 2.8. The theorem follows by the combination of Propositions 7.1 and 7.5.

\section{Appendix}

In this section we list some elementary results that are used at various places in the main body of the paper.

Lemma A.1. For $\delta>0, T \geq 0$, and $\omega \in C_{S}$,

$$
I_{\delta}(\omega)(\cdot \wedge T)=I_{\delta}(\omega(\cdot \wedge T)),
$$

and for all $\omega \in C_{S}$ such that $F_{\gamma}(\omega) \neq \underline{0}$, we have

$$
F_{\gamma}(\omega)(\cdot \wedge T)=F_{\gamma}(\omega(\cdot \wedge T))
$$

Lemma A.2. Let $\delta>0, T \geq 0, \omega \in C_{S}$ such that

$$
\omega(T) \in S_{2 \delta}^{0} \cup\left(S \backslash S_{\delta}\right),
$$

where $S_{2 \delta}^{0}$ is the interior of $S_{2 \delta}$. Then

$$
I_{\delta}(\omega(T+\cdot))(t)=I_{\delta}(\omega)(T+t)-I_{\delta}(\omega)(T), \quad t \geq 0 .
$$

Lemma A.3. For every sequence $\gamma \in \Gamma, T \in[0, \infty)$, and $\omega \in C_{S}$ such that $F_{\gamma}(\omega) \neq \underline{0}$, we have

$$
F_{\gamma}(\omega(T+\cdot))=F_{\gamma}(\omega)(T+\cdot)-F_{\gamma}(\omega)(T) .
$$


The relationship (70) can be cast in the form

$$
F_{\gamma}(\omega)(t)-F_{\gamma}(\omega)(s)=F_{\gamma}(\omega(s+\cdot))(t-s), \quad 0 \leq s \leq t .
$$

That is, $F_{\gamma}$ is additive on $\left\{\omega \in C_{S}: F_{\gamma}(\omega \neq \underline{0}\}\right.$. The combination of (70) and (67) yields that for $0 \leq T_{1} \leq T_{2}, \omega \in C_{S}$, and $\{\gamma(n), n \geq 1\} \in \Gamma$ such that $F_{\gamma}(\omega) \neq \underline{0}$, we have

$$
F_{\gamma}\left(\omega\left(\left(T_{1}+\cdot\right) \wedge T_{2}\right)\right)(t)=F_{\gamma}(\omega)\left(\left(T_{1}+t\right) \wedge T_{2}\right)-F_{\gamma}(\omega)\left(T_{1}\right) .
$$

Lemma A.4. Let $a, \lambda>0,\{\gamma(n), n \geq 1\} \in \Gamma$ and $\omega \in C_{S}$ be such that $F_{\gamma}(\omega) \neq \underline{0}$. Then

$$
a F_{\gamma}(\omega)(\lambda \cdot)=F_{a \gamma}(a \omega(\lambda \cdot)),
$$

where $a \gamma=\{a \gamma(n), n \geq 1\}$.

Lemma A.5. Suppose that $(z, y) \in \mathcal{A}$ and $T>0$ are such that $z(s) \neq 0$ for $s \in(0, T)$. Then, letting $u=\left(u_{1}, u_{2}\right)$ be the non-decreasing function from Definition 4.8 , we have that for $t \in[0, T]$,

$$
V_{1}(y,[0, t])=\left\|v_{1}\right\|\left(u_{1}(t)-u_{1}(0)\right)+\left\|v_{2}\right\|\left(u_{2}(t)-u_{2}(0)\right) .
$$

For the sake of brevity we omit the proofs of the above lemmas.

\section{References}

[1] J. Bertoin. Subordinators: Examples and applications. In Lectures on Probability Theory and Statistics (Saint-Flour, 1997) 1-91. Lecture Notes in Math. 1717. Springer, Berlin, 1999. MR1746300

[2] R. Blumenthal and R. Getoor. Markov Processes and Potential Theory. Academic Press, New York, 1968. MR0264757

[3] J. Bretagnolle. $p$-variation de fonctions aléatoires lère partie: Séries de Rademacher. In Séminaire de Probabilités VI, Université de Strasbourg 51-63. Springer, Berlin, 1972. MR0373019

[4] A. Brown and C. Pearcy. Introduction to Operator Theory. Springer, New York, Heidelberg, Berlin, 1977. MR0511596

[5] K. Burdzy and E. Toby. A Skorohod-type lemma and a decomposition of reflected Brownian motion. Ann. Probab. 23 (1995) $586-604$. MR1334162

[6] H. Chen and D. D. Yao. Fundamentals of Queueing Networks: Performance, Asymptotics, and Optimization. Stochastic Modelling and Applied Probability 46. Springer Science \& Business Media, New York, 2013. MR1835969

[7] V. Chistyakov and O. Galkin. On maps of bounded $p$-variation with $p>1$. Positivity 2 (1998) 19-45. MR1655755

[8] E. Çinlar. Probability and Stochastics. Graduate Texts in Mathematics 261. Springer, New York, Dordrecht, Heidelberg, 2011. MR2767184

[9] E. Çinlar, J. Jacod, P. Protter and M. Sharpe. Semimartingales and Markov processes. Probab. Theory Related Fields 54 (1980) 161-219. MR0597337

[10] J. W. Cohen and O. J. Boxma. Boundary Value Problems in Queueing System Analysis, 79. Elsevier, New York, 2000. MR0703000

[11] F. Coquet, A. Jakubowski, J. Mémin and L. Słominski. Natural decomposition of processes and weak Dirichlet processes. In In Memoriam Paul-André Meyer 81-116. Springer, Berlin, 2006. MR2276891

[12] J. Dai. Steady-state analysis of reflected Brownian motions: Characterization, numerical methods and queueing applications, Stanford University, 1990. MR2685786

[13] A. Dieker and J. Moriarty. Reflected Brownian motion in a wedge: Sum-of-exponential stationary densities. Electron. Commun. Probab. 15 (2009) 1, 1-16. MR2472171

[14] S. Ethier and T. Kurtz. Markov Processes: Characterization and Convergence. John Wiley \& Sons, New York, 1986. MR0838085

[15] G. Fayolle and R. Iasnogorodski. Two coupled processors: The reduction to a Riemann-Hilbert problem. Z. Wahrsch. Verw. Gebiete 47 (1979) 325-351. MR0525314

[16] M. Foddy. Analysis of Brownian motion with drift, confined to a quadrant by oblique reflection, Stanford University, 1983. MR2633392

[17] H. Föllmer. Calcul d'Itô sans probabilités. Sémin. Probab. Strasbourg 15 (1981) 143-150. MR0622559

[18] H. Föllmer. Dirichlet processes. In Stochastic Integrals 476-478. Springer, Berlin, 1981.

[19] W. Kang and K. Ramanan. A Dirichlet process characterization of a class of reflected diffusions. Ann. Probab. 38 (2010) $1062-1105$. MR2674994

[20] W. Kang and K. Ramanan. On the submartingale problem for reflected diffusions in domains with piecewise smooth boundaries. Preprint, 2014. Available at arXiv:1412.0729. MR3601653

[21] I. Karatzas and S. E. Shreve. Brownian Motion and Stochastic Calculus. Springer Science \& Business Media, New York, 1998. MR1121940

[22] E. Lieb and M. Loss. Analysis. American Mathematical Society, Providence, 2001. MR1817225

[23] K. Ramanan. Reflected diffusions defined via the extended Skorokhod map. Electron. J. Probab. 11 (2006) 934-992. MR2261058 
[24] K. Ramanan and M. I. Reiman. Fluid and heavy traffic diffusion limits for a generalized processor sharing model. Ann. Appl. Probab. 13 (2003) 100-139. MR1951995

[25] D. Revuz and M. Yor. Continuous Martingales and Brownian Motion. Springer, New York, 1999. MR1725357

[26] K. Sato. Lévy Processes and Infinitely Divisible Distributions. Cambridge University Press, Cambridge, 1999. MR1739520

[27] T. Simon. Small ball estimates in p-variation for stable processes. J. Theoret. Probab. 17 (2004) 979-1002. MR2105744

[28] S. Varadhan and R. Williams. Brownian motion in a wedge with oblique reflection. Comm. Pure Appl. Math. 38 (1985) 405-443. MR0792398

[29] R. Williams. Recurrence classification and invariant measure for reflected Brownian motion in a wedge. Ann. Probab. 13 (1985) 758-778. MR0799421

[30] R. Williams. Reflected Brownian motion in a wedge: Semimartingale property. Z. Wahrsch. Verw. Gebiete 69 (1985) 161-176. MR0779455

[31] R. Williams. Local time and excursions of reflected Brownian motion in a wedge. Publ. Res. Inst. Math. Sci. 23 (1987) 297-319. MR0890921 